\title{
Monitorización Y Control De Calidad De Las Estaciones De La Red CGPS Topo-Iberia-UJA
}

\author{
Juan Antonio García-Armenteros, \\ DEA en Ingeniería Geodésica y Cartográfica, Candidato a Doctor, \\ Servicio GNSS, CEACTierra, Universidad de Jaén, España
}

URL:http://dx.doi.org/10.19044/esj.2020.v16n24p1

\section{Resumen}

La medición de la deformación de la corteza terrestre utilizando técnicas basadas en Sistemas Globales de Navegación por Satélite (GNSS) fue una de las metodologías a utilizar en el proyecto Topo-Iberia. Concretamente se utilizó el Sistema de Posicionamiento Global (GPS) y se estableció una red de veintiséis estaciones operando continuamente (cGPS) en la Península Ibérica y Norte de África. Las redes GNSS requieren un control permanente para asegurarse de que las estaciones funcionan correctamente y que la calidad de sus datos es apropiada para estudios geodinámicos. En este trabajo se ha desarrollado y presentado por primera vez la monitorización y control de calidad de las estaciones de la red Topo-Iberia que están a cargo de la Universidad de Jaén. Se han descrito los procesos del control de calidad implementados en el Servicio GNSS del CEACTierra, abarcando desde la recuperación de datos GPS de las estaciones desplegadas en campo hasta su envío al Instituto Geológico y Minero de España (IGME), institución encargada de centralizarlos. Se ha estudiado la calidad de un periodo de casi once años en el caso de estaciones activas y se han analizado los eventos acontecidos más significativos. El estudio manifiesta que los emplazamientos de estas estaciones son óptimos para conformar una red geodésica y que los valores en sus parámetros de calidad están al nivel de las mejores estaciones del IGS. Además, este estudio complementa la labor del IGME proporcionando información sobre sus datos GPS recopilados que hasta ahora no existía.

Palabras clave: Control de calidad, Cordillera Bética, monitorización automática, red cGPS, Topo-Iberia 


\title{
Monitoring and Quality Control of the Topo-Iberia-UJA CGPS Network Stations
}

\author{
Juan Antonio García-Armenteros, \\ DEA of Geodesy and Cartography Engineering, PhD candidate, \\ GNSS Service, CEACTierra, University of Jaén, Spain
}

\begin{abstract}
Measurement of crustal deformation using Global Navigation Satellite System (GNSS)-based techniques was one of methodologies used in the TopoIberia project. Global Positioning System (GPS) was used, and a network of twenty-six continuously operating stations (cGPS) was established on the Iberian Peninsula and in North Africa. GNSS networks require continuous control to ensure that the stations work properly and data quality is appropriate for geodynamic studies. The monitoring and quality control of the Topo-Iberia network stations, being under the charge of the University of Jaén, are developed and presented for the first time in this paper. The quality control processes implemented at the CEACTierra GNSS Service are described, ranging from retrieving GPS data from stations deployed in the field to uploading the data to the Spanish Geological Survey (IGME), the institution in charge of storing them. The quality of a period of almost eleven years is studied in the case of active stations, and the most significant events analyzed. The study shows that the locations of these stations are optimal to form a geodetic network and the values in its quality parameters are on a par with the best IGS stations. Furthermore, this study complements the IGME work by providing information on its collected GPS data that previously did not exist.
\end{abstract}

Keywords: Quality control, Betic Cordillera, automatic monitoring, cGPS network, Topo-Iberia

\section{Introducción}

Las redes de estaciones permanentes GNSS (Global Navigation Satellite System) permiten obtener posicionamientos con precisiones centimétricas, e incluso milimétricas. Una de sus aplicaciones en el ámbito de la geodinámica es la medición de la deformación cortical. Ejemplo de ello es el despliegue de redes permanentes internacionales a escala global que permite estudiar los desplazamientos de placas tectónicas y sus movimientos relativos. La evolución de las redes, su implantación a escala nacional, regional y su densificación ha hecho posible estudiar desplazamientos debido a fallas 
activas, subsidencias, terremotos, vulcanismo, etc., incluso a escala local. Con este fin se desplegó dentro del proyecto Topo-Iberia (Gallart, 2006) una red de veintiséis estaciones en la Península Ibérica y norte de África complementando las redes ya existentes. En concreto las estaciones se equiparon con receptores capacitados para registrar observaciones en el Sistema de Posicionamiento Global y operando continuamente (cGPS) (Figura 1).

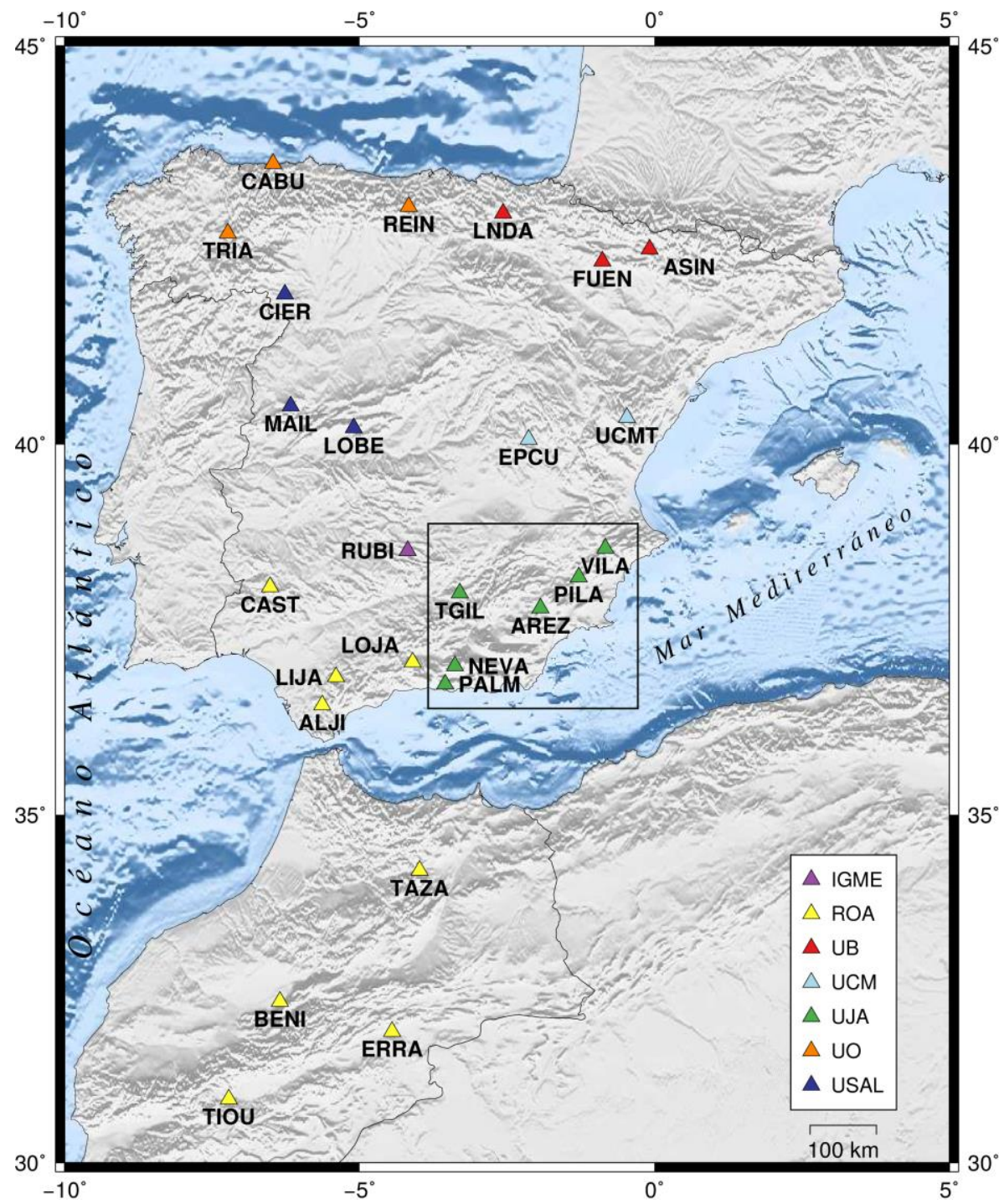

Figura 1. Despliegue de las veintiséis estaciones cGPS de la red Topo-Iberia en función de la institución a cargo. El rectángulo indica la zona de estudio. Relieve sombreado extraído del Modelo de Relieve Global de ETOPO1 (Amante y Eakins, 2009), iluminación desde el sur. Figura creada mediante el software Generic Mapping Tools (GMT) (Wessel et al., 2013) 
El Instituto Geológico y Minero de España (IGME) implementó el Sistema de Información de Topo-Iberia (SiTopo) donde se almacenan los datos de esa red. El mantenimiento y control de las estaciones está repartido entre algunas de las instituciones que formaban el equipo Consolider TopoIberia, entre ellas la Universidad de Jaén (Tabla 1).

Tabla 1. Estaciones cGPS del proyecto Topo-Iberia. Cuatro caracteres de identificación (ID), posición geográfica y localización, fecha de activación e institución a cargo (IGME: Instituto Geológico y Minero de España; ROA: Real Instituto y Observatorio de la Armada;

UB: Universidad de Barcelona; UCM: Universidad Complutense de Madrid; UJA:

Universidad de Jaén; UO: Universidad de Oviedo; USAL: Universidad de Salamanca). Posición geográfica tomada de Gárate et al. (2015)

\begin{tabular}{|c|c|c|c|c|c|}
\hline ID & Lon. $\left({ }^{\circ}\right)$ & at. $\left({ }^{\circ}\right)$ & Localización & Fecha & Inst. \\
\hline JI & 94 & & ádi & & 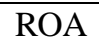 \\
\hline EZ & & & ก̃a & & UJA \\
\hline & & & A sín & & UB \\
\hline ENI & & & $\cos$ & & ROA \\
\hline $\mathrm{ABU}$ & 00 & & le Cabo E & & UO \\
\hline AST & -6.5321 & & o de Seg & & ROA \\
\hline IER & -6.2807 & & Irdeciervos - Zamora, Es & & USA \\
\hline PCU & -2.1 & & $\mathrm{Cu}$ & & $\mathrm{UCM}$ \\
\hline$A$ & -4.4 & 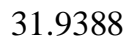 & & & ROA \\
\hline $\mathrm{EN}$ & & & & & UB \\
\hline & & & & & ROA \\
\hline & & & & & UB \\
\hline BE & & & ña & & JSAI \\
\hline JA & -4.1 & & & & ROA \\
\hline AIL & & & & & USA \\
\hline EVA & & & & & UJA \\
\hline & & & Gra & & UJA \\
\hline & -1.2 & & $a{ }^{2}$ & & UJA \\
\hline & & & & & UO \\
\hline & & & The & & IGM \\
\hline ZA & & & & & ROA \\
\hline & & & & & UJA \\
\hline & & & & & ROA \\
\hline & & & & /08 & UO \\
\hline MT & 22 & & & $15 / 07 / 08$ & UCM \\
\hline LA & -0.8 & 05 & Sierra de la Villa, Villena - Alicante, España & $22 / 09 / 08$ & UJA \\
\hline
\end{tabular}

Los datos registrados en esas estaciones son procesados posteriormente mediante software científico de alta precisión, como Bernese GNSS Software, GAMIT/GLOBK o GipsyX, obteniendo series temporales y mediante su análisis se determinan los vectores de deformación de la corteza terrestre, el campo de velocidades. Ello permite monitorizar deformaciones milimétricas producidas por la convergencia entre las placas tectónicas África y Eurasia, además de deformaciones intraplaca, así como identificar áreas y 
fallas sísmicas específicas que presenten variaciones en las velocidades de deformación, lo que podría implicar un incremento de riesgo sísmico en dichas zonas. El proceso completo se recoge en Gárate et al. (2015) en el cual la red Topo-Iberia fue analizada con estos tres softwares por tres grupos pertenecientes a diferentes instituciones. Se procesaron los primeros 4 años de actividad de la red con diferentes estrategias estimando la deformación de la corteza terrestre en la Península Ibérica y Marruecos. Previo al procesado se realizó un control de calidad de los datos GPS necesario para detectar datos anómalos, pero no derivó en un estudio sobre la calidad de las estaciones de la red.

Los objetivos del presente trabajo son realizar el estudio de la calidad de las estaciones de la red Topo-Iberia que la Universidad de Jaén tiene a su cargo, la red Topo-Iberia-UJA, analizando la estabilidad y calidad temporal tanto de las estaciones como de los datos observados y verificando que sean óptimos para su uso siguiendo recomendaciones del International GNSS Service (IGS), mostrar los beneficios de la monitorización remota diaria y la posibilidad de implementarse en toda la red. Un ejemplo de este tipo de control diario son los realizados por servicios internacionales como EUREF Permanent GNSS Network (EPN) en EUREF (2020) y University NAVstar COnsortium (UNAVCO) sobre la red PBO (Plate Boundary Observatory) en UNAVCO (2020).

El despliegue de la red Topo-Iberia se realizó a lo largo de diversas transectas y zonas estratégicas de la Península Ibérica y norte de África. El control de calidad objeto del presente trabajo se pretende realizar sobre las estaciones AREZ, PALM, PILA, NEVA, TGIL y VILA, por tanto, la zona de estudio se corresponde con su zona de despliegue, la Cordillera Bética Oriental en el sur de la Península Ibérica. En esa zona, dentro del complejo contexto general de extensión y desplazamientos hacia el oeste de las Béticas, existe una tectónica activa moderada de carácter compresivo (Palano et al., 2013, Galindo-Zaldivar et al., 2015), en donde se acomoda parte de la convergencia entre Nubia y Eurasia mediante acortamiento (Borque et al., 2019).

\section{Metodología}

\subsection{Control de calidad de las estaciones}

La calidad de una red GNSS permanente con fines geodésicos no depende exclusivamente de los equipos instalados, sino también de la estabilidad del monumento que los soporte y de la ubicación física de la estación. Por lo general, los equipos de las estaciones Topo-Iberia se instalaron sobre el terreno y las antenas en pilares de hormigón anclados sobre afloramientos rocosos, siguiendo criterios recomendados por UNAVCO (Gárate et al., 2008). Los equipos constan de receptores de doble frecuencia Trimble modelo NetRS con antenas GNSS Trimble Choke-Ring. Se utilizaron 
radomos protectores de antena $\mathrm{SCIGN}$ solo en aquellos lugares donde las condiciones ambientales lo aconsejaran, como en la estación NEVA donde se instaló la versión corta (Tabla 2). La alimentación eléctrica se realiza mediante paneles solares y dos baterías, y la comunicación con la estación vía módem GPRS.

Tabla 2. Estaciones cGPS bajo el control de la UJA. Cuatro caracteres de identificación (ID), fecha de la primera y última medición del periodo controlado, duración, días usados en el análisis y modelos de antena y receptor instalados

\begin{tabular}{lccccccc}
\hline ID & $\begin{array}{c}\text { Fecha } \\
\text { inicial }\end{array}$ & $\begin{array}{c}\text { Fecha } \\
\text { final }\end{array}$ & $\begin{array}{c}\text { Duración } \\
\text { (años) }\end{array}$ & Días & Antena GNSS & Radomo & Receptor GPS \\
\hline AREZ & 2008.3 & 2018.8 & 10.5 & 3818 & TRM29659.00 & NONE & Trimble NetRS \\
NEVA & 2008.8 & 2019.2 & 10.4 & 3680 & TRM29659.00 & SCIS & Trimble NetRS \\
PALM & 2008.5 & 2019.2 & 10.7 & 3929 & TRM29659.00 & NONE & Trimble NetRS \\
PILA & 2008.5 & 2019.2 & 10.7 & 3929 & TRM29659.00 & NONE & Trimble NetRS \\
TGIL & 2008.3 & 2017.8 & 9.5 & 3420 & TRM29659.00 & NONE & Trimble NetRS \\
VILA & 2008.7 & 2009.7 & 1.0 & 348 & TRM29659.00 & NONE & Trimble NetRS \\
\hline
\end{tabular}

El control de la calidad de las estaciones asignadas a la Universidad de Jaén se realizó una vez implementada la red reflejando los resultados en el trabajo de investigación García-Armenteros (2010). Consistió en el análisis de diversos factores relacionados con: la presencia de obstáculos en el horizonte de la estación, efectos de reflexión de la señal, potencia e intensidad de la señal recibida, relación señal-ruido (SNR), número de observaciones realizadas, etc. Para evaluar los obstáculos que puedan impedir la recepción de la señal se utilizó el software libre QC2SKY (Roggero, 2010) que representa gráficamente los ficheros del control de calidad generados por el software TEQC (Translate/Edit/Quality Check) (Estey y Meertens, 1999) desarrollado por UNAVCO. TEQC es un software de procesamiento de observaciones GNSS que proporciona información de la calidad de los datos como son los valores de SNR, efecto multitrayectoria, saltos de ciclo, y retardo ionosférico. QC2SKY representa el gráfico del cielo en función de la elevación y azimut del satélite en el momento de la observación en dos diferentes sistemas de coordenadas, Cartesiano y Polar. De este modo se manifiestan los obstáculos existentes y su proyección en este sistema de coordenadas. Para el estudio del efecto multitrayectoria en cada una de las frecuencias L1 y L2 se establece una proyección cenital de las trayectorias de los satélites observados, y mediante un código de color (Figura 2) se distinguen en función del acimut las zonas donde las reflexiones de la señal son mayores.

El efecto multitrayectoria o interferencias que pudiera ocasionar el entorno de la estación suele ser de carácter permanente y característico del lugar. Como consecuencia los gráficos del cielo en días consecutivos resultan similares, de modo que es suficiente un ciclo completo de la constelación GPS 
para obtener el horizonte de oclusión de las estaciones y detectar graves problemas de señal si los hubiera. Además, las estaciones están configuradas para registrar observaciones cada 30 segundos. Por todo ello el análisis se realizó sobre 24 horas de observación y con dicho intervalo de muestreo. La fecha de observación corresponde al día de puesta en funcionamiento de cada estación. Se requiere una máscara de $0^{\circ}$ en el ángulo de elevación para obtener una visión general de todas las observaciones sobre el horizonte y la información completa sobre las barreras u oclusiones presentes.

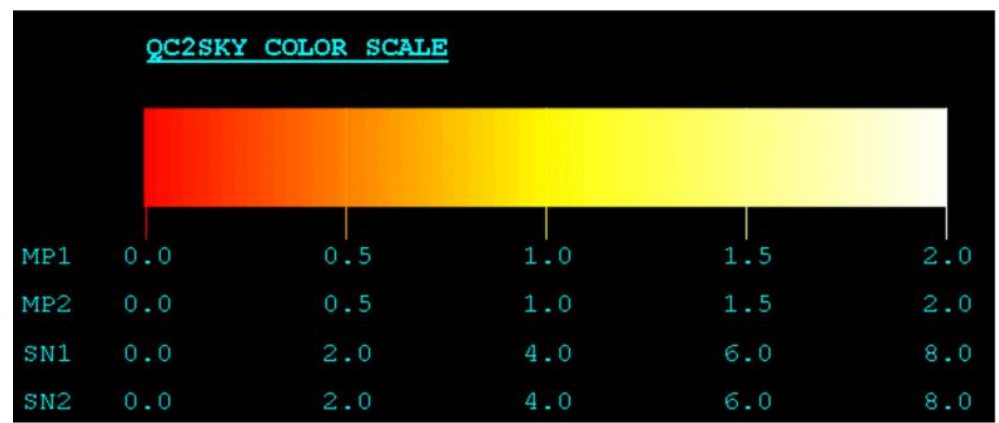

Figura 2. Escala cromática utilizada en el software QC2SKY. Valores en metros. Imagen tomada y modificada de Roggero (2010)

\subsection{Control de calidad de los datos}

Diariamente los datos descargados de las estaciones cGPS a cargo de la UJA son sometidos a un control de la calidad muy similar al realizado por EUREF (Carpentier et al., 2004) utilizando el software TEQC. Previamente, los datos descargados en formato binario nativo son transformados a formato RINEX v2.11 (Receiver Independent Exchange Format Version 2.11) y posteriormente son editados y sometidos al control de calidad con TEQC en modo qc (quality control). Los ficheros de entrada son los RINEX diarios de observación y navegación, y el fichero de salida es un pequeño informe en el que una línea resumen (SUM) contiene diversas variables (Figura 3) en las cuales se basa el control de calidad: \#expt como número de observaciones esperadas, \#have como número de observaciones registradas completas, \% como ratio entre los dos anteriores (\#have/\#expt), mp1 y mp2 como raíz cuadrática media (RMS) del efecto multitrayectoria en L1 y L2 respectivamente, y o/slps como el número de observaciones por salto de ciclo. Al incluir el fichero de navegación, el control de la calidad realizado es el denominado completo «qc-full» y los estadísticos resultantes corresponden por defecto a una máscara de $10^{\circ}$ en el ángulo de elevación, aunque las estaciones estén configuradas para seguir todos los satélites por encima de $0^{\circ}$. 
first epoch last epoch

Figura 3. Ejemplo de línea SUM del informe generado por TEQC conteniendo los parámetros en los que se basa el control de calidad

Los parámetros diarios de calidad obtenidos permiten controlar la calidad de las observaciones de las estaciones, es decir, monitorizar su comportamiento a lo largo del tiempo. Esa información se muestra en gráficas anuales que se componen de:

- Máximo número de observaciones: Indica el número de observaciones completas reales que fueron contabilizadas para cada frecuencia sobre el horizonte y por encima de la máscara en el ángulo de elevación. Actualmente en TEQC, para la constelación GPS, una «observación completa» es tener ambos observables, pseudodistancia y fase, en la frecuencia L1 y en al menos otra frecuencia para un satélite (Estey, 2018). Este parámetro depende del número de épocas y en este caso, al ser treinta segundos el intervalo de registro, resulta en 2878 épocas.

- Ratio observaciones registradas/esperadas: El ratio o/e es el porcentaje del número de observaciones completas registradas con respecto al número de observaciones esperadas o posibles. El número de observaciones esperadas asume un registro completo de datos sobre la máscara de elevación de $10^{\circ}$ establecida en el control de calidad y asume un intervalo de registro de épocas constante. Además, solo es posible para aquellos satélites con efemérides conocidas por lo que es necesario el fichero de navegación.

- $\quad$ RMS del efecto multitrayectoria en L1 y L2: Representa la raíz media cuadrática en metros del efecto multitrayectoria para las dos frecuencias GPS (L1 y L2) promediada sobre el intervalo de observación. La variable MP1 estudiada es el RMS de la combinación lineal de la medida de código y fase en L1 con la medida de fase en L2, y MP2 es el RMS de la combinación lineal de la medida de código y fase en L2 con la medida de fase en L1. El efecto multitrayectoria se debe a que la amplitud y fase de la señal GPS se ve afectada por su reflexión sobre la superficie en torno a la antena (árboles, edificios, rocas, cobertura vegetal, etc.). Las señales directa y reflejada se suman y como consecuencia se incrementa la distancia aparente desde el satélite causando un error en el posicionamiento.

- Número de saltos de ciclo: Indica los saltos de ciclo por cada 1000 observaciones completas (parámetro o/slps en Figura 3 invertido y multiplicado por 1000). Se debe a una pérdida de captura de señal en los datos de fase o código. En TEQC salto de ciclo significa salto de ciclo en el retardo ionosférico (IOD) de la fase y/o salto para uno o 
ambos efectos multitrayectoria de código (MP1 y MP2), ocurridos durante la época que se tiene una observación completa para un satélite en particular. Las causas pueden ser variadas: baja elevación del satélite, presencia de obstáculos físicos, bajo SNR, mal funcionamiento del receptor, etc.

Las estaciones del International GNSS Service son el referente seguido para determinar qué son «buenos» valores en la calidad de los datos (IGS, 2017). Una estación observando durante 24 horas en intervalos de 30 segundos resultará en 20000 o más observaciones para la constelación GPS. La mitad de las estaciones IGS tienen valores por debajo de 5, 0.4 y 0.6 en saltos de ciclo, RMS MP1 y RMS MP2 respectivamente (representado con línea horizontal verde en las gráficas de calidad). Y dos tercios de las estaciones IGS tienen valores por debajo de 10, 0.5 y 0.75 también para esos parámetros (representado con línea horizontal naranja).

Las gráficas de calidad se complementan con información procedente de los «site log». Estos son registros normalizados de los metadatos de cada estación, siguiendo la estructura establecida en IGS (2011), donde además se recopilan todos los cambios o actualizaciones producidos en el equipamiento (cambio de antena, receptor, firmware, etc.) y que son representados en las gráficas mediante líneas verticales.

\subsection{Software y flujo de datos}

El control de calidad expuesto es solamente una de las fases a las que los datos son sometidos y era necesario automatizarlo de modo que permitiera monitorizar remotamente las estaciones a diario. Los datos almacenados en las estaciones pasan por diferentes fases: descarga, conversión, preprocesado, control de calidad y creación de gráficas, compactación, compresión, almacenamiento y envío al IGME. En todos esos procesos se ha utilizado numeroso software procurando que sea gratuito, estable y con soporte a largo plazo. El sistema operativo elegido fue la distribución Debian GNU/Linux (SPI, 2020) en su versión «estable», recomendada para aplicaciones que requieren un alto nivel de estabilidad y seguridad. También se buscó que el software fuese ejecutable por líneas de comando, ya que para gestionar todos esos procesos y tareas se pretendía desarrollar una serie de programas o scripts almacenados en ficheros de texto plano y ejecutables en consola o shell. Los códigos se escribieron en lenguaje de programación Bash (Ramey, 2020) combinado con otros lenguajes como Gawk (FSF, 2016) y Python (PSF, 2020), que ejecutan a su vez otros programas que intervienen en las diferentes fases.

Cada uno de los procesos, desde que los datos son descargados de las estaciones en campo hasta que finalmente son enviados al IGME, son controlados por tres scripts. A continuación, se describen los procesos que 
cada uno de ellos realizan sucesivamente. Un script denominado «control_topoiberia_uja.sh»realiza automáticamente la mayoría de las fases del control diario:

- $\quad$ Descarga de ficheros en formato Trimble *.T00 vía Internet y GPRS (General Packet Radio Service) de los servidores de las estaciones cGPS, mediante el cliente ftp (File Transfer Protocol) LFTP (Lukyanov, 2016).

- Conversión de ficheros *.T00 en formato *.dat y *.mes para que puedan ser leídos por TEQC mediante Trimble runpkr00 (Trimble, 2016).

- Conversión de ficheros *.dat en formato RINEX v2.11 de observación xxxxddds.yyo y de navegación xxxxddds.yyn y su posterior edición mediante TEQC.

- Control de calidad «qc-full» de los RINEX mediante TEQC y obtención del informe de calidad *.S.

- Creación del registro de parámetros de calidad y en caso de incidencias notificación en pantalla.

- Compactación y compresión de los RINEX siguiendo los estándares internacionales mediante rnx2crx (Hatanaka, 2008) y (N)compress (Jannesen, 2016).

- Subida de datos al servidor del IGME mediante LFTP.

El script «plot_teqc_sum.sh»produce todas las gráficas de calidad a partir de los registros de parámetros obtenidos anteriormente. Durante su ejecución crea otro código que es ejecutado por Gnuplot (Williams y Kelley, 2016), programa de visualización gráfica de datos científicos. Y con el script «qc_cal.sh»se crea un calendario en formato XLS informando de la disponibilidad de RINEX diarios y de su calidad mediante una escala cromática en función de uno de los parámetros de calidad controlados (número de épocas, observaciones registradas, MP1, etc.). El flujo de datos GPS desde las estaciones en campo hasta el IGME, fases descritas y scripts que las controlan dentro del Servicio GNSS se muestran en la Figura 4. 


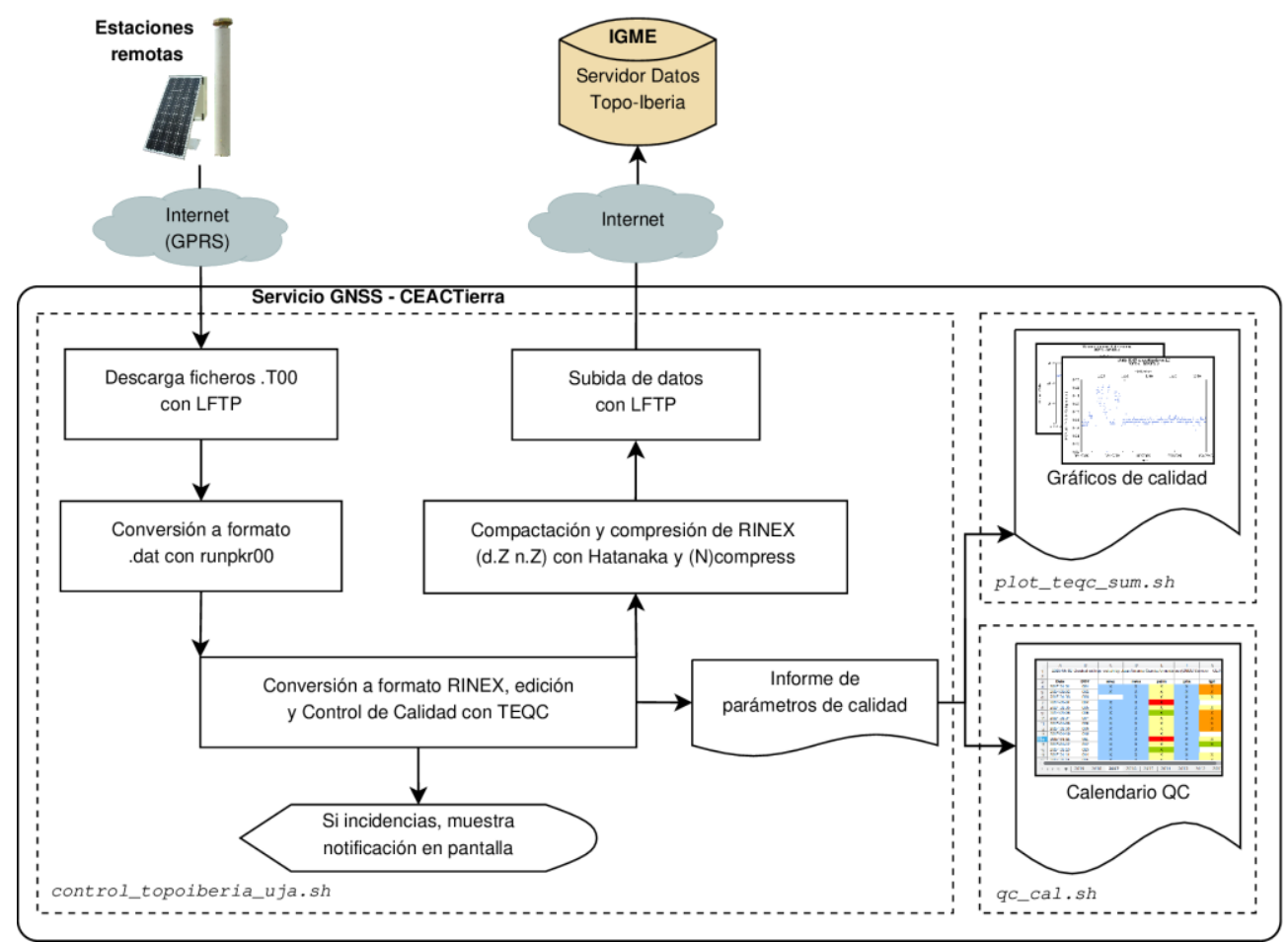

Figura 4. Flujo de datos dentro del Servicio GNSS y procesos agrupados según el script que los controla

RINEX v2.11 es considerado el estándar para el intercambio de datos GNSS en el formato RINEX 2. El hecho de que las estaciones de la red TopoIberia registren observaciones en el sistema GPS, manteniendo este formato, permite el uso de TEQC para realizar el control de calidad. Redes más modernas registran observaciones en otros sistemas satelitales (GLONASS, Galileo, SBAS, BeiDou, QZSS, IRNSS, etc.) y usan el formato RINEX v3.x, expresamente diseñado para acomodarse al incremento y complejidad de las nuevas constelaciones GNSS y nuevo estándar recomendado por el IGS. TEQC no puede trabajar con este nuevo formato, aunque con previa conversión a formato RINEX v2.11 aún es posible hacerlo incorporando una versión extendida de RINEX v2.11 (Estey, 2013; Estey, 2017), formato no reconocido por el IGS. Actualmente UNAVCO no da soporte para TEQC y no se espera que haya nuevas actualizaciones que admita el formato RINEX 3 y aprovechar todas las características de las nuevas señales GNSS. De producirse en el futuro la modernización de la red Topo-Iberia, permitiendo observaciones multi-GNSS y usando el formato RINEX 3, el proceso del control de calidad no se vería afectado notablemente, ya que en vez de TEQC se usaría el software G-Nut/Anubis (Vaclavovic y Dousa, 2016) desarrollado por Geodetic Observatory Pecný (GOP). Este software es también una 
herramienta ejecutable por línea de comando para realizar el control de calidad, pero en este caso para todas las constelaciones GNSS disponibles, con el que se obtendrían los parámetros de calidad integrándose en el flujo de datos expuesto.

\section{Resultados y discusión}

\subsection{Calidad de las estaciones}

En la Figura 5 se muestran algunos gráficos del cielo obtenidos con QC2SKY para cada una de las estaciones en función de la elevación y azimut de los satélites registrados el día de su activación. Los saltos de ciclo son trazados con círculos verdes. En el sistema de coordenadas Cartesiano se observa que el horizonte de oclusión en las estaciones se encuentra por debajo de los $10^{\circ}$ no habiendo importantes obstáculos, excepto en el caso de NEVA y PALM. En NEVA se distingue un obstáculo en dirección sureste que coincide con el Pico Veleta (Sierra Nevada), y en PALM el obstáculo identificado en dirección oeste corresponde al Pico Guindalera (Sierra de los Guájares), ambos en Granada. Aun así, esas dos estaciones poseen un horizonte suficientemente amplio como para registrar las observaciones necesarias, hecho que se comprobó al realizar el control de calidad de los datos. Respecto al efecto multitrayectoria y SNR no se identifican en ninguna estación valores anómalos que indiquen un posible problema en sus ubicaciones. También se muestra un ejemplo de ambos parámetros para las estaciones NEVA y PALM.

\subsection{Calidad de los datos}

Las gráficas multianuales o históricas (Figuras 6.A - 6.E) muestran la calidad de los datos en las estaciones bajo el control de la UJA desde su activación en 2008 y corresponden a una media de 10 años de datos continuos para cada estación, excepto VILA (Tabla 2). Independientemente de que los valores de estas estaciones permanentes se consideren buenos tomando como referencia las estaciones IGS, es útil buscar cambios significativos en el seguimiento de la estación. Esto ayudaría a detectar y resolver posibles problemas que pudieran ocurrir e incluso comprender la causa en cambios posicionales que se determinen a partir de los datos. En parámetros como el efecto multitrayectoria, característico del entorno de la estación por la topografía del terreno y obstáculos que pudiera haber, es más interesante observar si sufre cambios bruscos o mantenidos en el tiempo. Así, en el caso de que no haya habido cambios en el equipamiento, podría indicar por ejemplo crecimiento de la cobertura vegetal o introducción de cualquier objeto por el hombre, y ayudar a tomar las medidas oportunas si fuese necesario. 

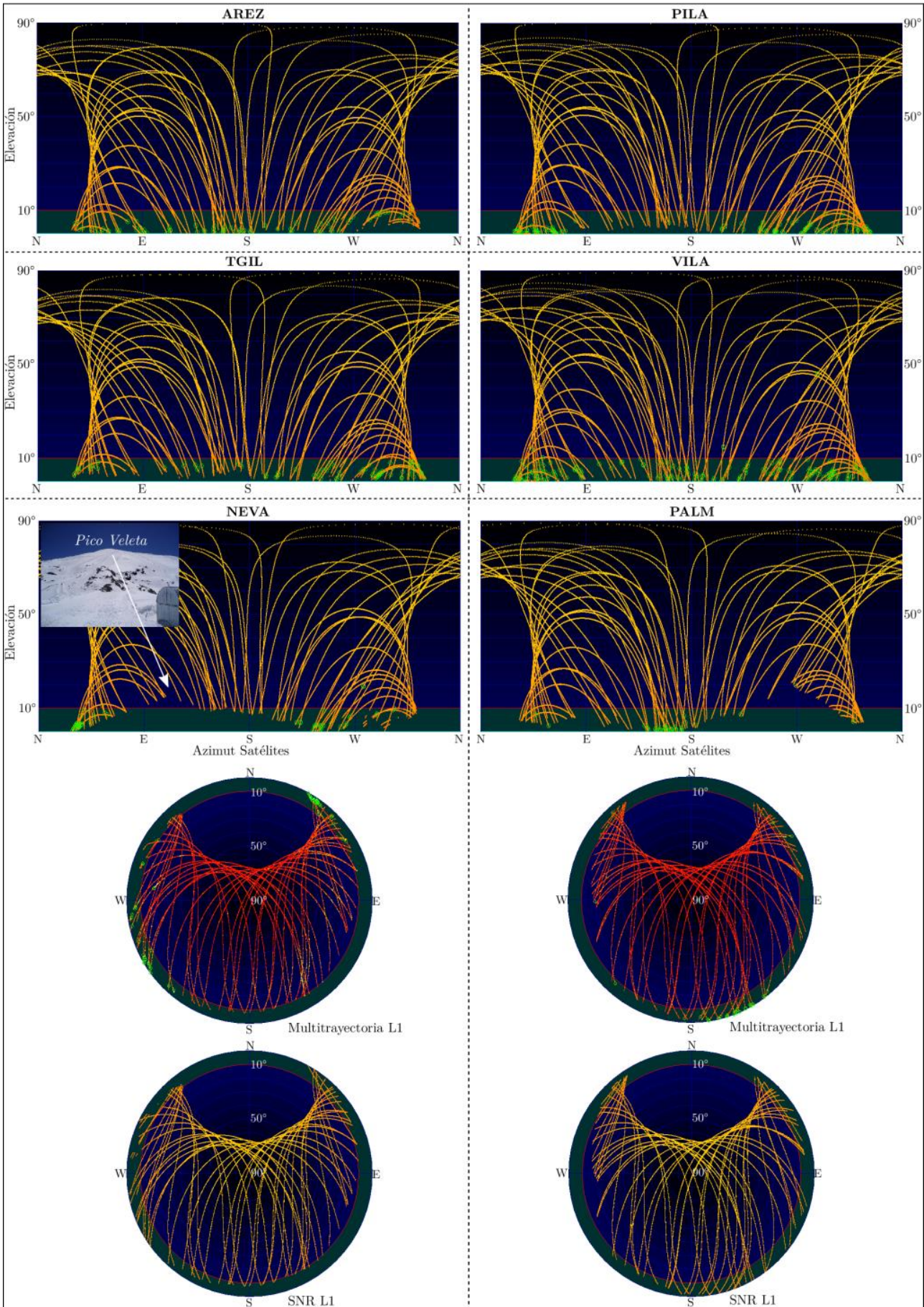

Figura 5. Gráficos del cielo en función de la elevación y azimut de los satélites desde cada estación en sistema de coordenadas Cartesiano. MP1 y SNR1 en sistema de coordenadas Polar 


\section{Control de Calidad GNSS/GPS Histórico}

Estación: AREZ - RINEX 2 Red: Topo-Iberia

Periodo: 2008-05-09 (DOY 130) / 2018-10-29 (DOY 302) Semana GPS: 1478 / 2025

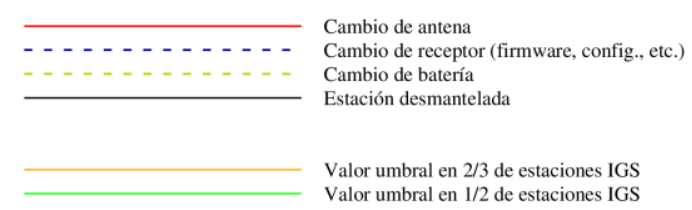

Máximo número de observaciones

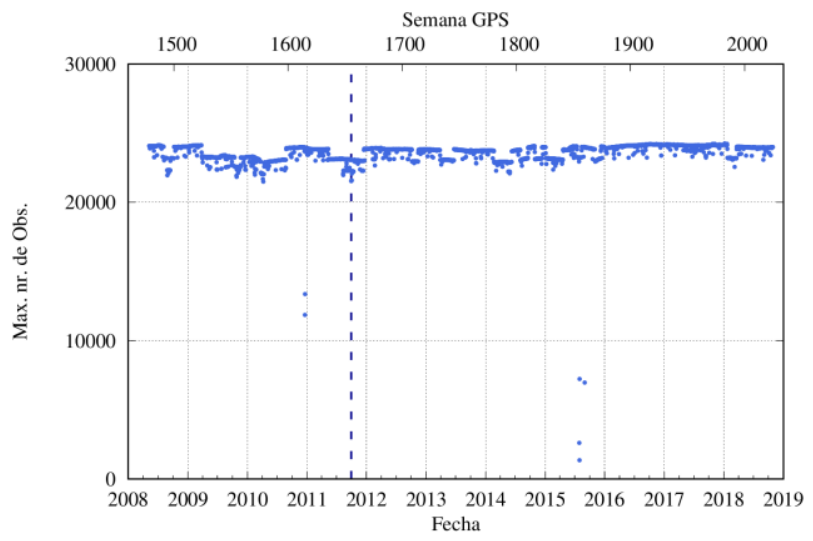

Ratio observaciones registradas/esperadas

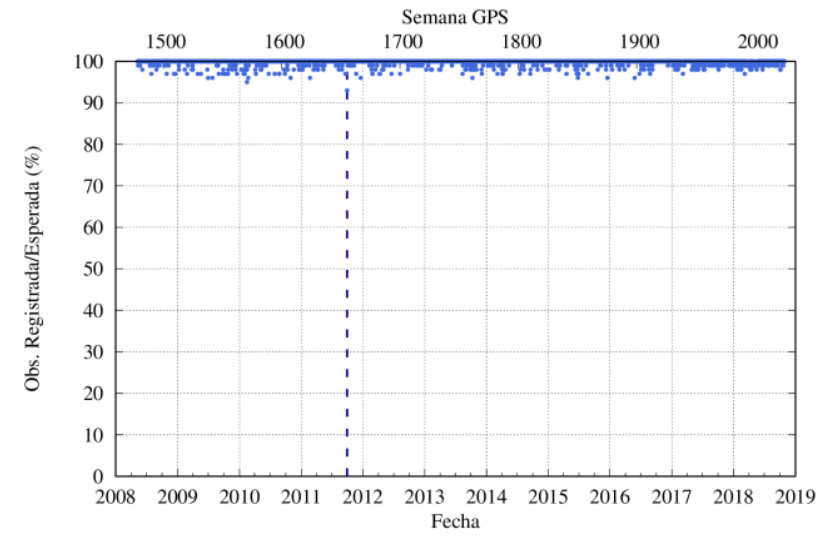

Número de saltos de ciclo

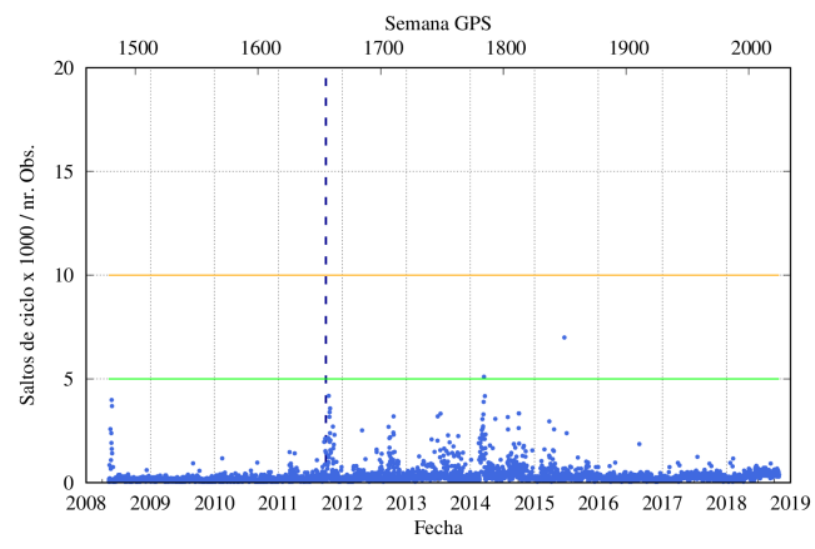

RMS diario del efecto multitrayectoria en $\mathrm{L1}$

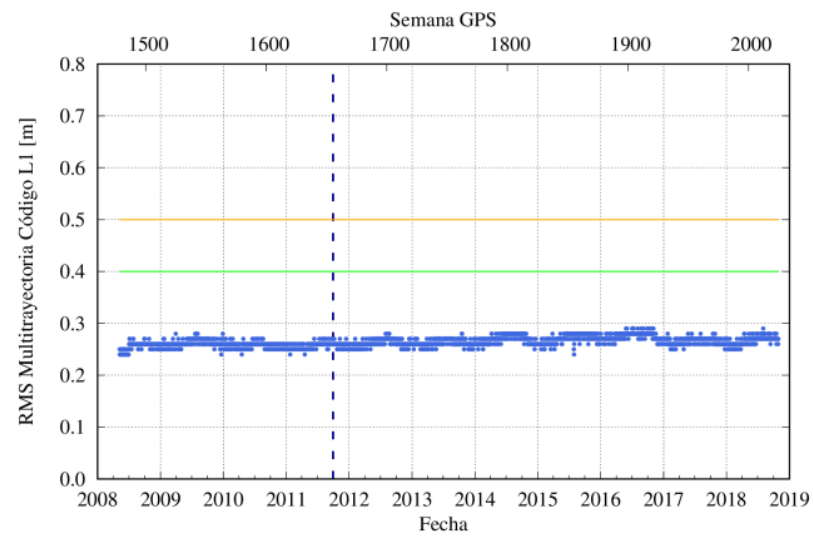

RMS diario del efecto multitrayectoria en L2

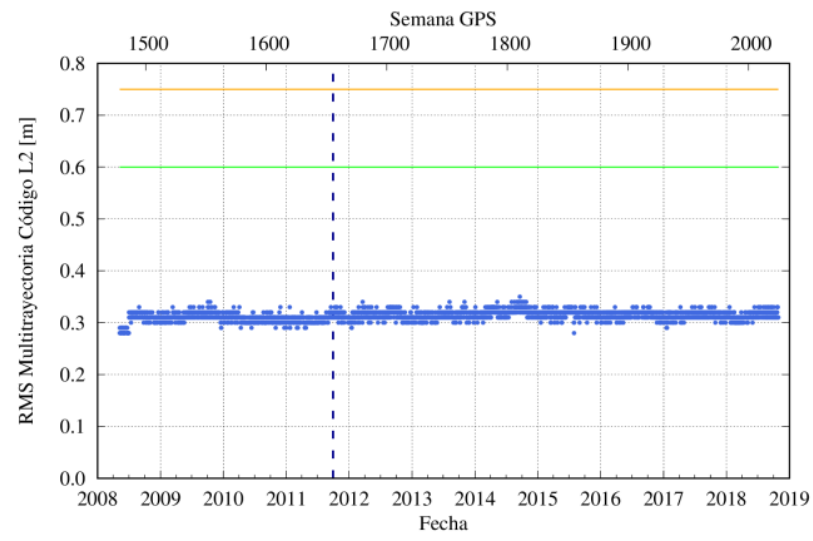

Figura 6.A. Información sobre la calidad de los datos históricos completos de la estación AREZ 


\section{Control de Calidad GNSS/GPS Histórico}

Estación: NEVA - RINEX 2 Red: Topo-Iberia

Periodo: 2008-11-01 (DOY 306) / 2019-03-31 (DOY 090) Semana GPS: 1503 / 2047

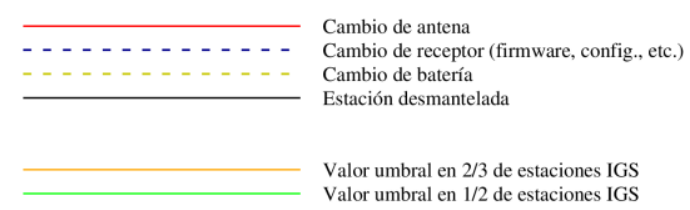

Máximo número de observaciones

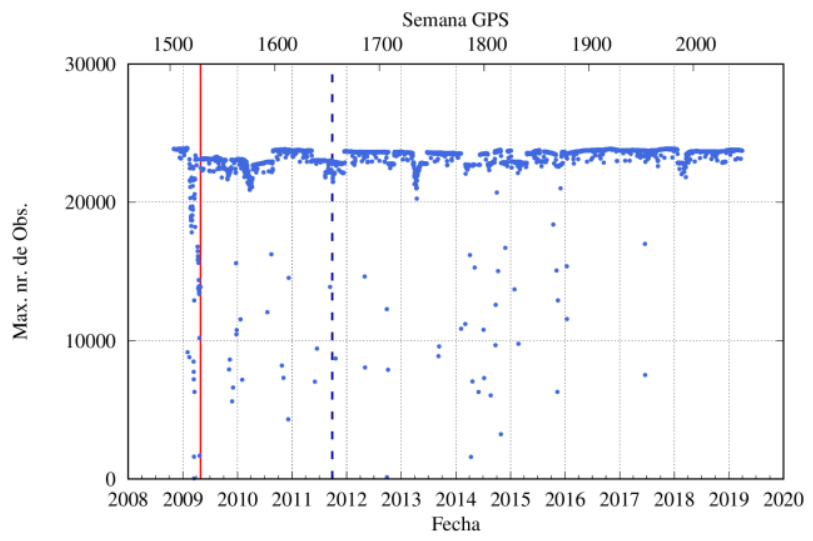

Ratio observaciones registradas/esperadas

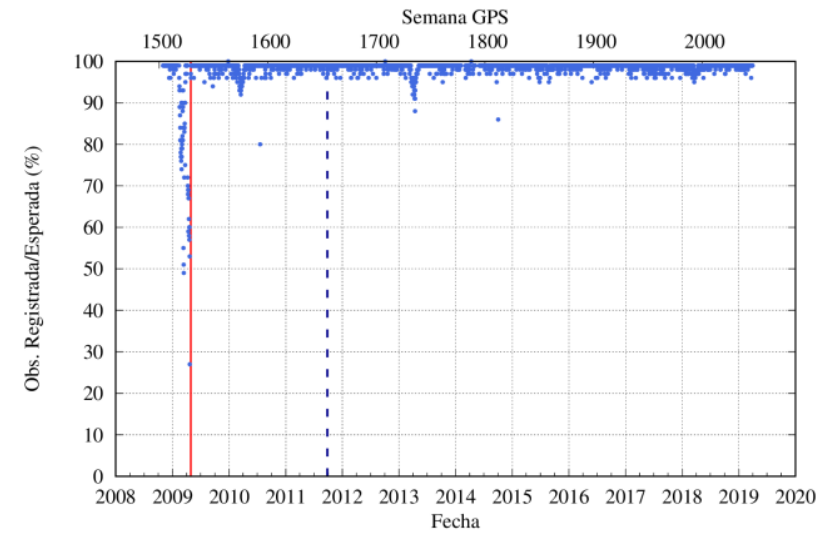

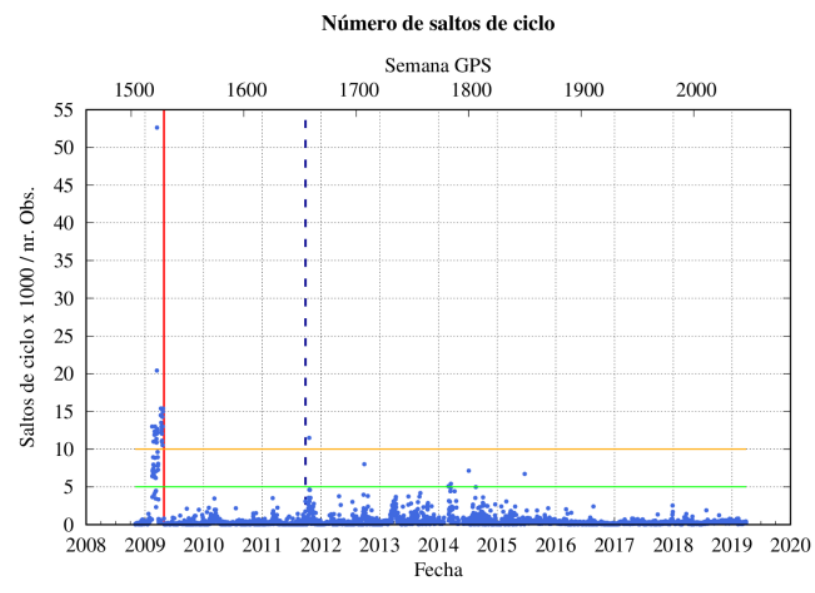

RMS diario del efecto multitrayectoria en $\mathrm{L1}$

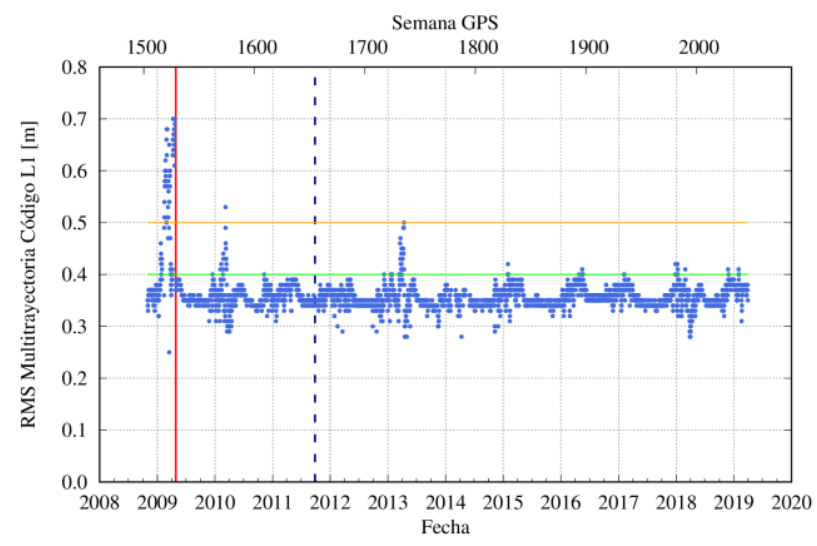

RMS diario del efecto multitrayectoria en L2

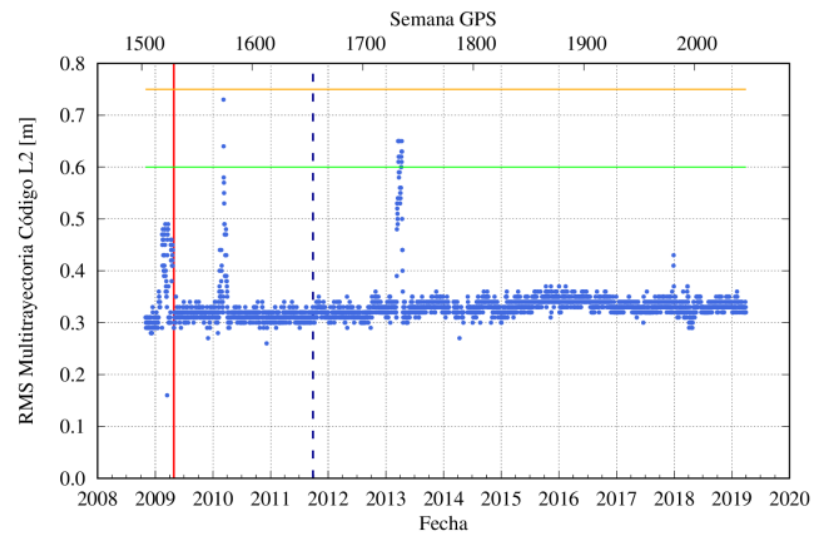

Figura 6.B. Información sobre la calidad de los datos históricos completos de la estación NEVA 


\section{Control de Calidad GNSS/GPS Histórico}

Estación: PALM - RINEX 2 Red: Topo-Iberia

Periodo: 2008-06-19 (DOY 171) / 2019-03-31 (DOY 090) Semana GPS: 1484 / 2047

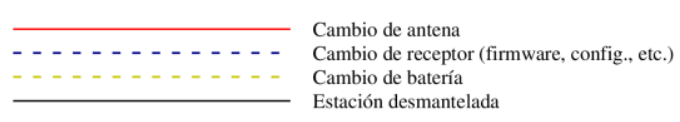

Valor umbral en 2/3 de estaciones IGS Valor umbral en $1 / 2$ de estaciones IGS

Máximo número de observaciones

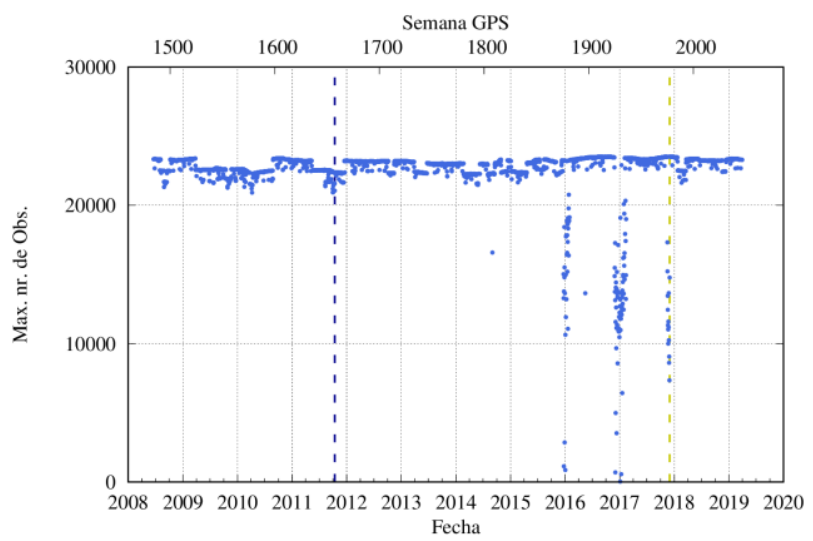

Ratio observaciones registradas/esperadas

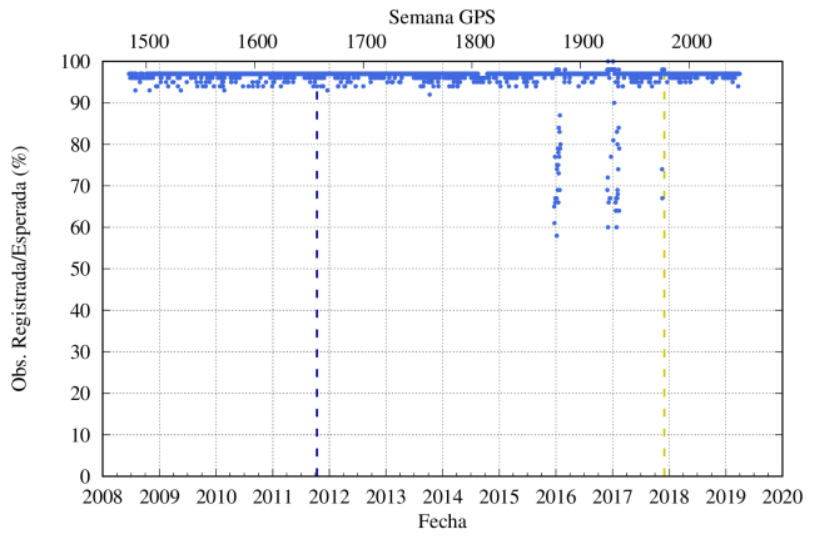

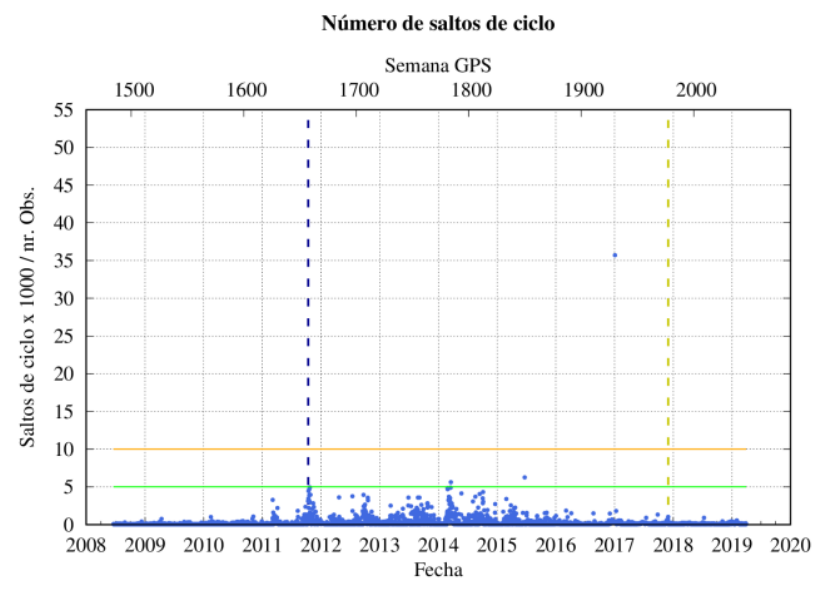

RMS diario del efecto multitrayectoria en $\mathrm{L1}$

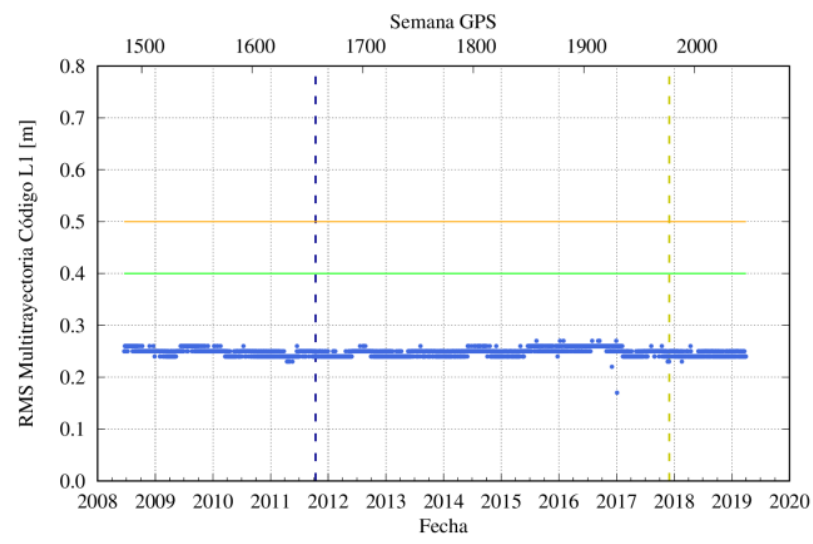

RMS diario del efecto multitrayectoria en L2

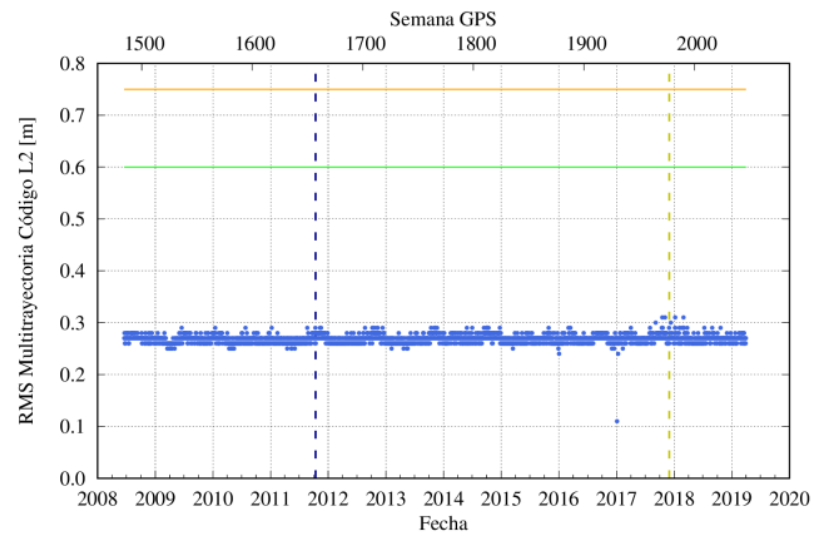

Figura 6.C. Información sobre la calidad de los datos históricos completos de la estación

\section{PALM}




\section{Control de Calidad GNSS/GPS Histórico}

Estación: PILA - RINEX 2 Red: Topo-Iberia

Periodo: 2008-06-27 (DOY 179) / 2019-03-31 (DOY 090) Semana GPS: 1485 / 2047

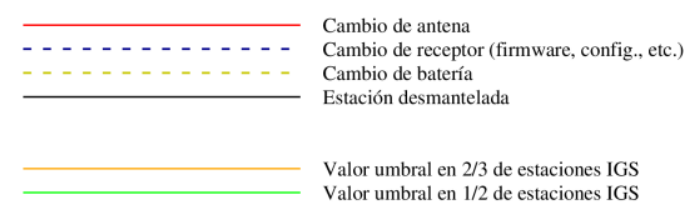

Máximo número de observaciones

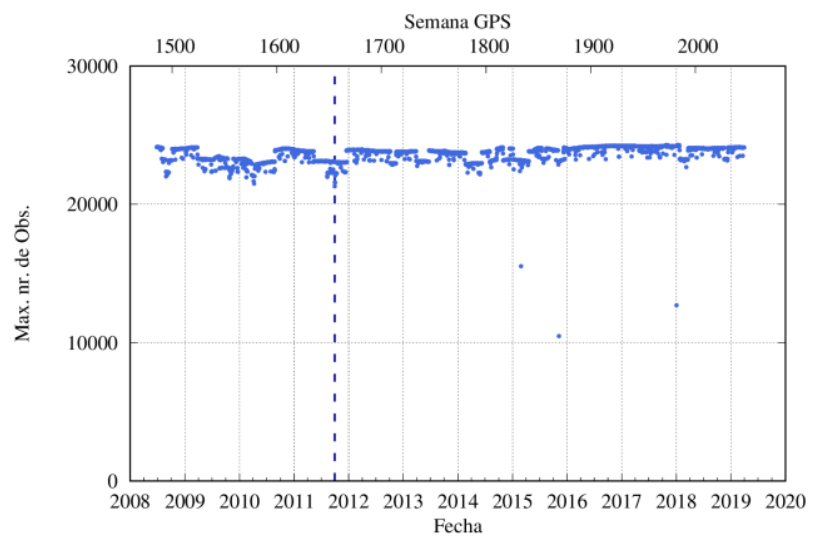

Ratio observaciones registradas/esperadas

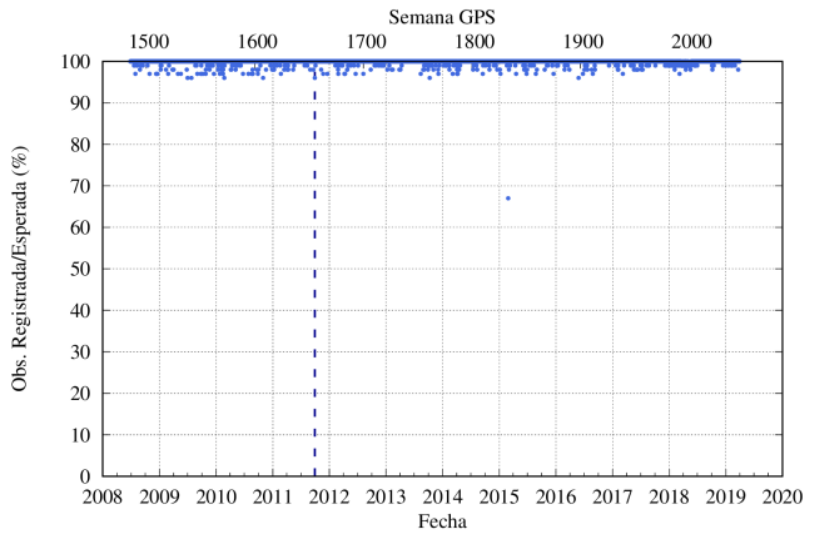

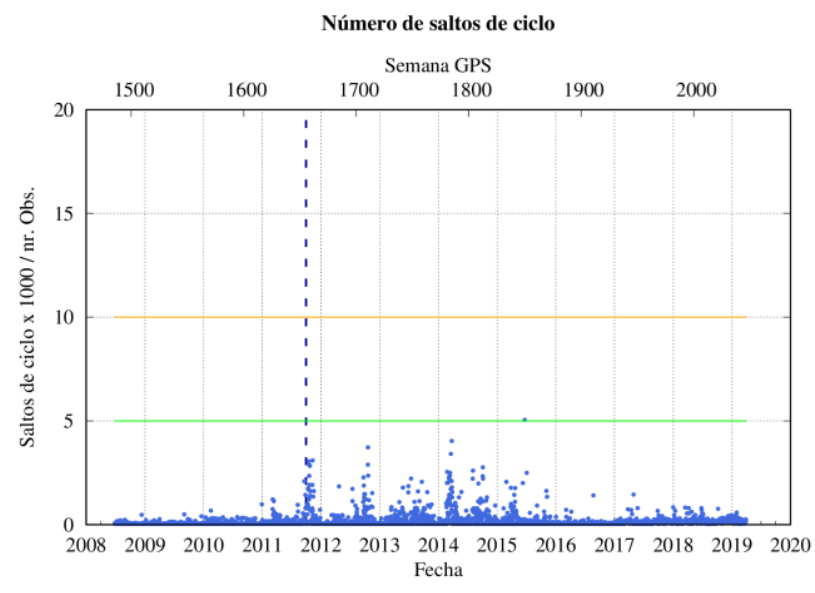

RMS diario del efecto multitrayectoria en $\mathrm{L1}$

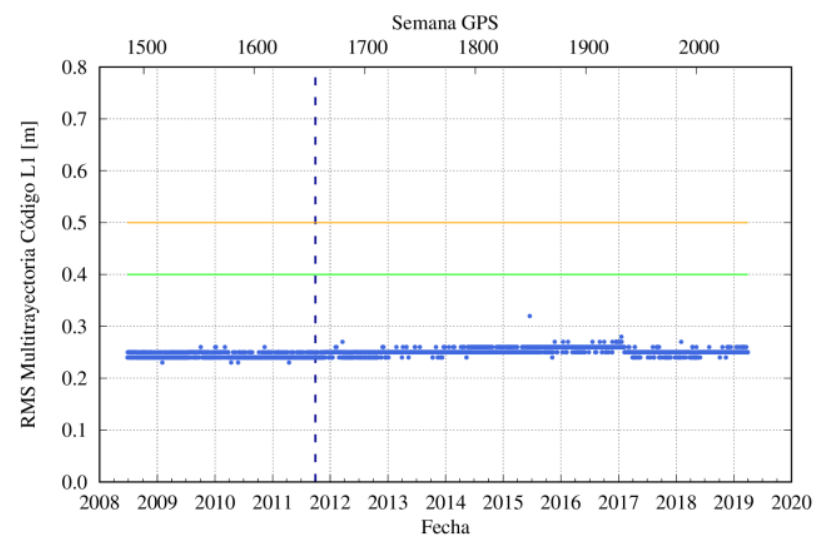

RMS diario del efecto multitrayectoria en L2

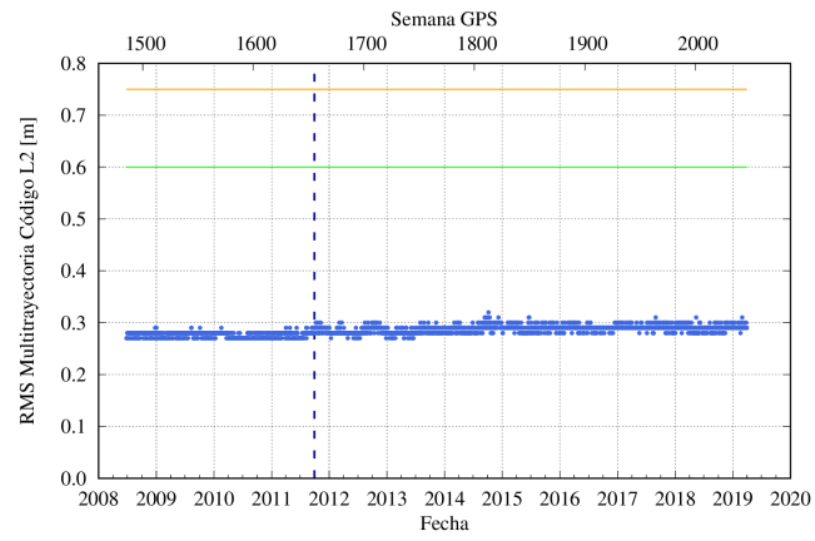

Figura 6.D. Información sobre la calidad de los datos históricos completos de la estación PILA 


\section{Control de Calidad GNSS/GPS Histórico}

Estación: TGIL - RINEX 2 Red: Topo-Iberia

Periodo: 2008-04-22 (DOY 113) / 2017-10-03 (DOY 276) Semana GPS: 1476 / 1969
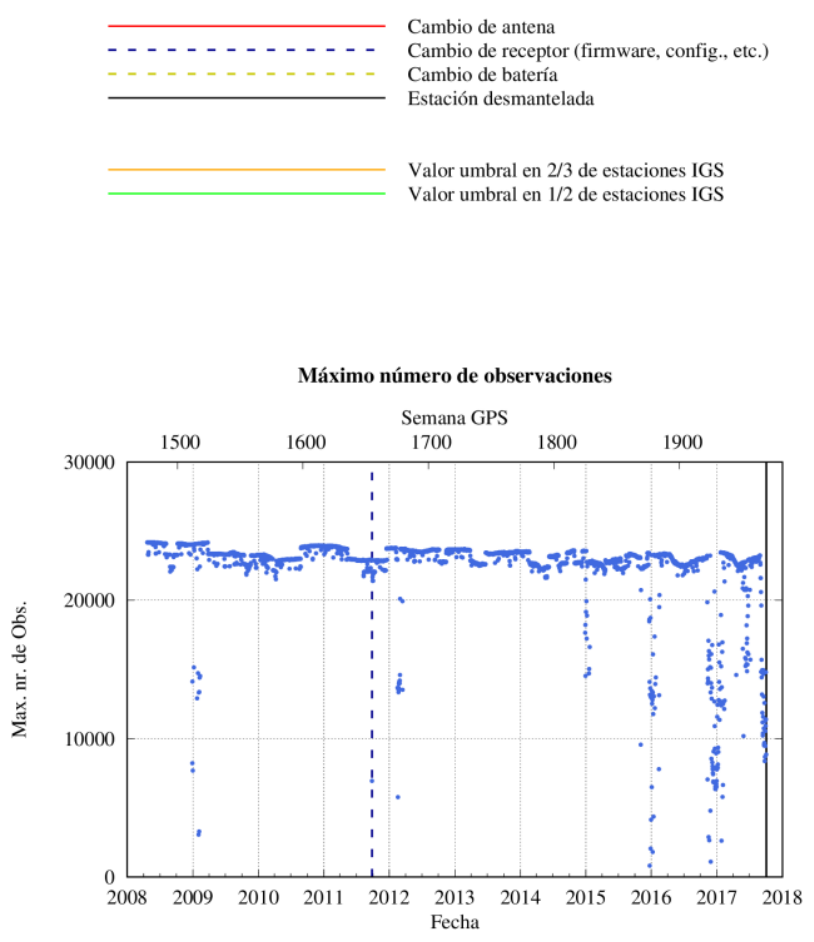

Ratio observaciones registradas/esperadas

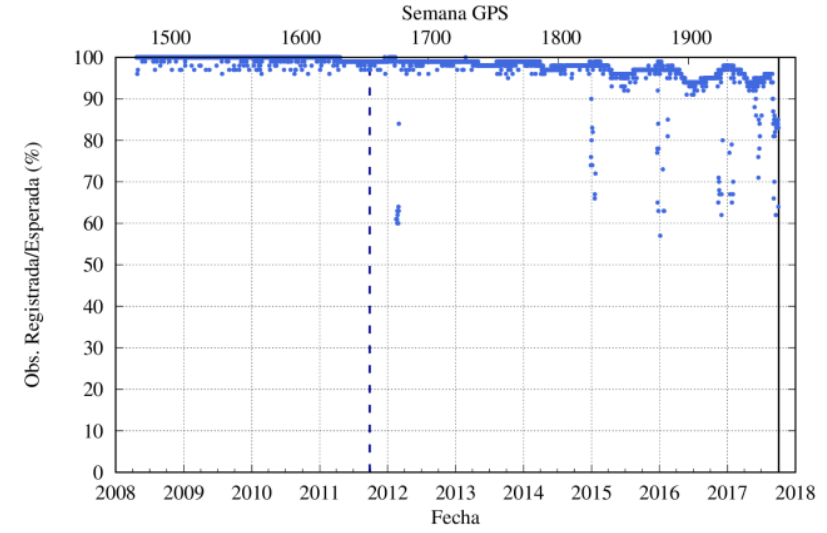

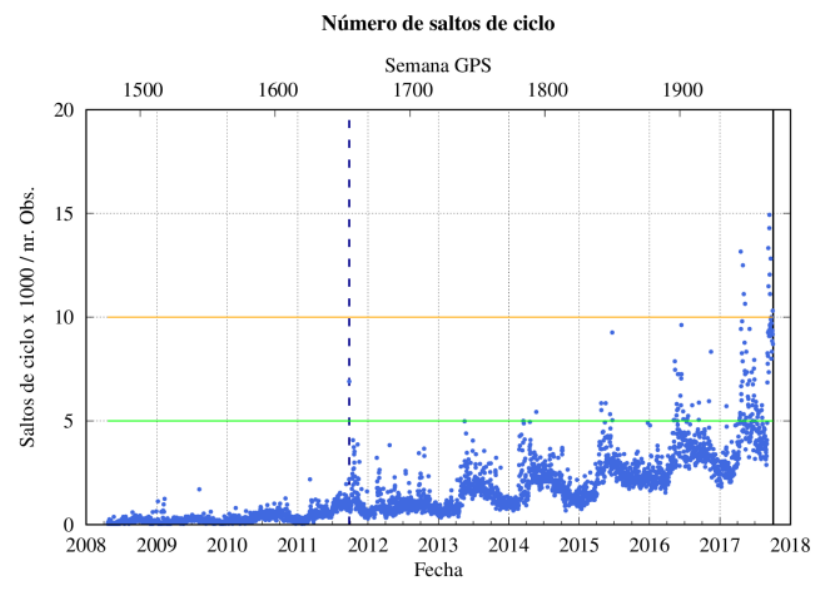

RMS diario del efecto multitrayectoria en L1

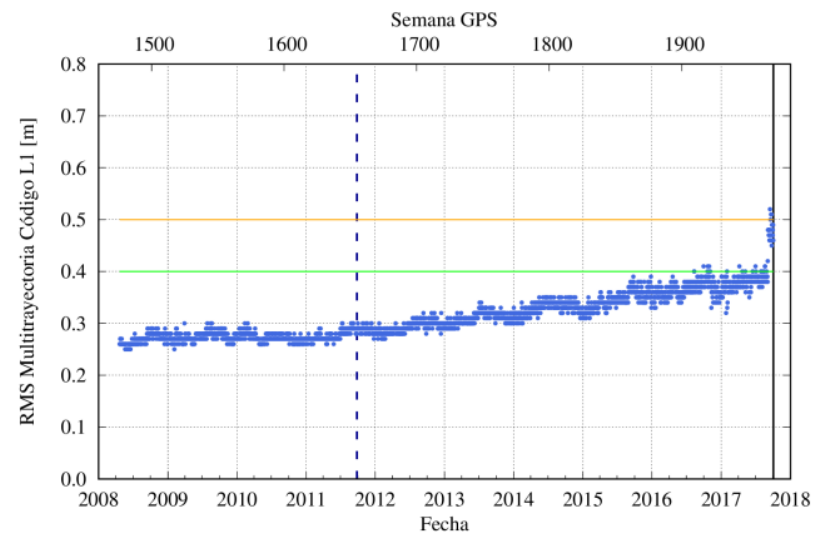

RMS diario del efecto multitrayectoria en L2

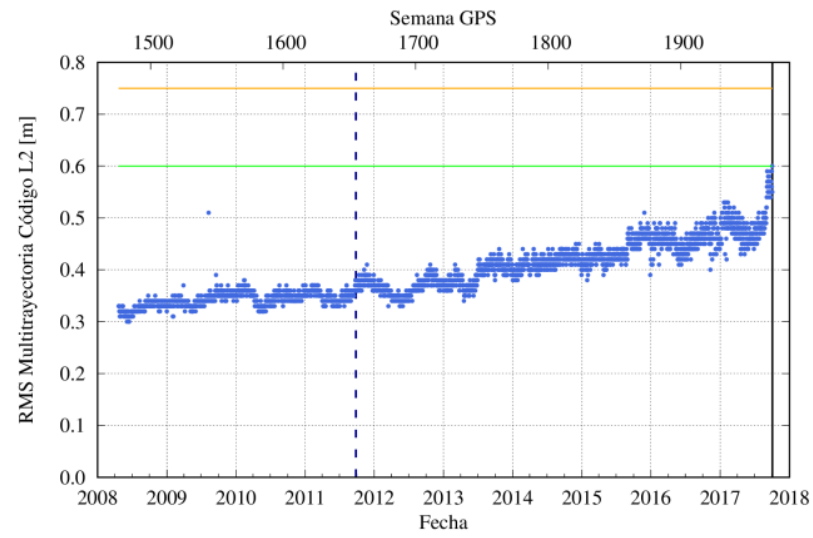

Figura 6.E. Información sobre la calidad de los datos históricos completos de la estación TGIL 


\subsubsection{Estación AREZ}

El funcionamiento de la estación (Figura 7) desde su activación en 2008 ha sido correcto y su calidad puede considerarse buena, teniendo como referente los valores umbrales de calidad en las estaciones IGS expuestos anteriormente, como muestra su gráfica de calidad histórica (Figura 6.A). Los parámetros de calidad analizados se han mantenido estables a lo largo de un periodo de actividad de 10.5 años, destacando el alto número de observaciones completas registradas y el ratio o/e cercano al $100 \%$. No ha habido periodos de tiempo sin registro de datos y las gráficas del efecto multitrayectoria y saltos de ciclo reflejan ausencia de anomalías. Ambos parámetros se han mantenido por debajo del umbral correspondiente al que poseen la mitad de las estaciones IGS (representado con línea horizontal verde), lo cual sitúa la calidad de AREZ al nivel de dichas estaciones.

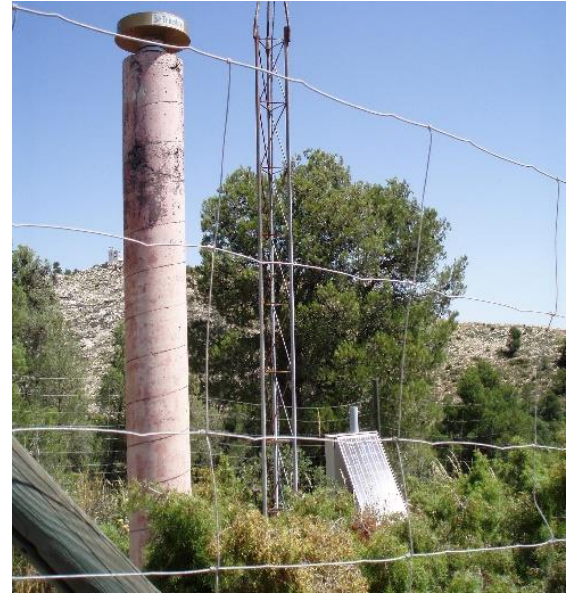

Figura 7. Estación GPS permanente AREZ en Sierra del Almirez, Lorca Murcia

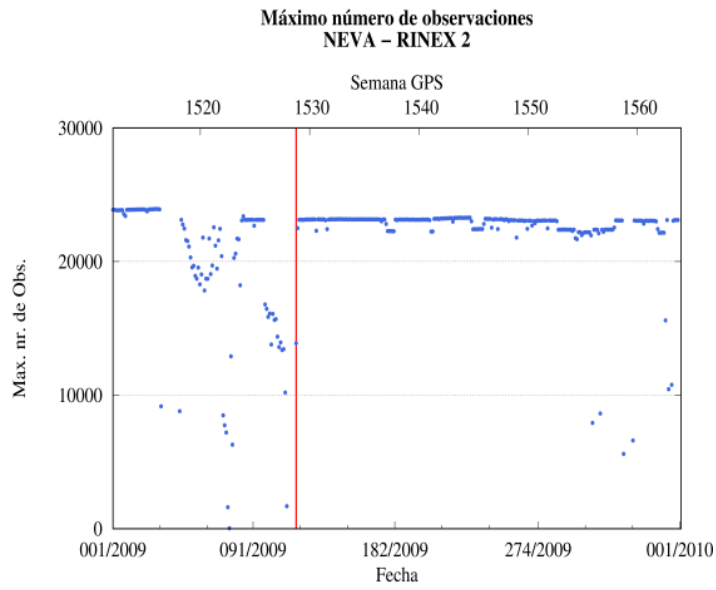

Figura 8. Número de observaciones completas registradas por la estación NEVA en 2009. Línea vertical roja indica un cambio de antena

\subsubsection{Estación NEVA}

Se aprecia en su gráfica de calidad histórica (Figura 6.B), y con más detalle en su gráfica anual (Figura 8), que a partir del 1 de febrero de 2009 (Day Of Year - DOY 032) comenzó a decrecer el número de observaciones completas habiendo incluso días sin registros. El hecho de que también se reflejen cambios bruscos en el resto de parámetros, aumentando los saltos de ciclo y efecto multitrayectoria en L1 y L2, evidenció un grave problema en la estación que se resolvió cambiando la antena el 29 de abril de 2009 - DOY 119 (indicado con línea roja sobre la gráfica). Los valores de RMS en MP1 y MP2 se mantuvieron bajos durante el resto de actividad de la estación, pero se aprecia una periodicidad coincidiendo con la época invernal. Ello se puede relacionar con la acumulación de nieve sobre el radomo de antena y su alrededor que aumenta la reflexión de la señal GPS (Figura 9). 


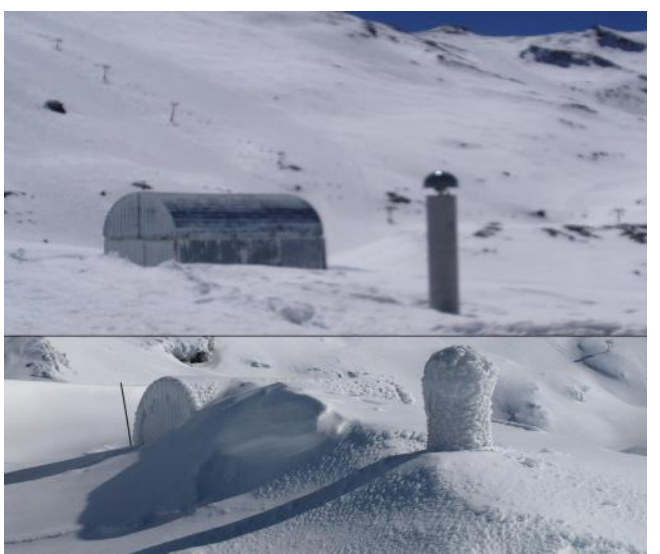

Figura 9. Estación GPS permanente NEVA en Sierra Nevada, Granada. La nieve cubre la antena con radomo y su entorno durante algunos meses produciendo variaciones en efecto multitrayectoria

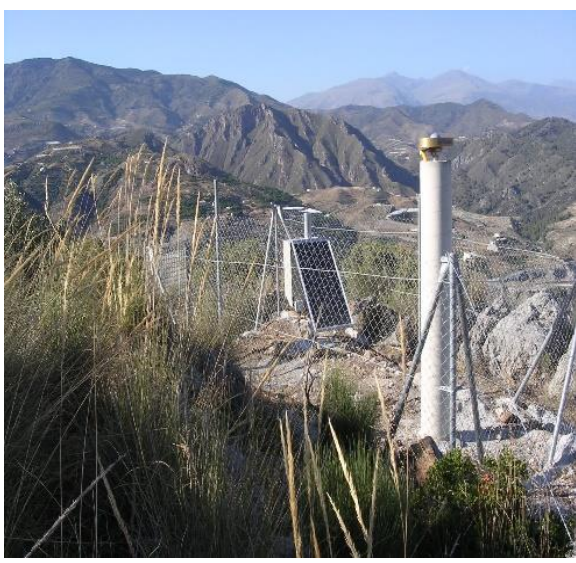

Figura 10. Estación GPS permanente PALM en Sierra de los Guájares,

Granada. Se distingue el panel solar junto al armario que contiene el receptor y baterías

Jaldehag et al. (1996) estudió el efecto de la acumulación de nieve y sus resultados indicaban que produce retardo de la señal y aumenta su dispersión, afectando a la coordenada vertical de la posición estimada. Además, las variaciones en el espesor de la nieve y efecto multitrayectoria están relacionados de tal forma que en Larson y Nievinski (2013) se demostró que las estaciones GPS instaladas con fines geodinámicos pueden ser usadas también para estimar espesores de nieve con una periodicidad diaria. Aunque esa periodicidad no se aprecia en el resto de parámetros de calidad, conviene tenerlo en cuenta cuando se estimen las posiciones durante esa época del año. También se distinguen varios días aislados con número reducido de observaciones completas sin una correspondencia asociada en el ratio o/e, siendo éste prácticamente constante y cercano al 100\%. Ello se debió a cortes en el suministro eléctrico de la estación. En general, la alimentación en las estaciones cGPS de la red Topo-Iberia se realiza mediante paneles solares y dos baterías, pero la estación NEVA está conectada directamente a la red eléctrica del Observatorio de Sierra Nevada.

\subsubsection{Estación PALM}

Al igual que en NEVA, se aprecia un descenso en el registro de observaciones completas coincidiendo con la época invernal, periodo 20152018 (Figura 6.C). En este caso no se corresponde con alteraciones continuadas en los saltos de ciclo y efecto multitrayectoria, los cuales se mantenían estables y con buenos valores a excepción de un día en enero de 2017 que alcanzó 36 saltos de ciclo. En cambio, se detecta una clara correspondencia entre el descenso de observaciones completas registradas y 
el ratio o/e. Si el comportamiento hubiera sido como en la estación NEVA, manteniéndose constante el ratio, se podría deducir un problema en la alimentación eléctrica, pero el descenso del ratio sugería a priori un mal funcionamiento. Por otro lado, en diciembre de 2017 el comportamiento fue similar al de NEVA, es decir, el funcionamiento era correcto pero las ventanas de observación eran cortas o nulas comenzando a registrar observaciones por la mañana. La ausencia de obstáculos en el entorno de la estación que produzcan zonas de sombra sobre el panel solar (Figura 10), evidenció un problema en las baterías que proporcionan energía a la estación. El panel solar proporcionaba la energía necesaria durante el día, pero este tiempo de actividad solar no era suficiente para recargar la batería y continuar durante la noche. Este problema se resolvió cambiando la batería el 1 de diciembre de 2017 - DOY 335 (indicado con línea vertical discontinua amarilla sobre el gráfico de calidad).

\subsubsection{Estación PILA}

El funcionamiento de la estación PILA (Figura 11) ha sido similar al de la estación AREZ desde su activación, como muestra su gráfica de calidad histórica (Figura 6.D). No se ha producido ningún evento que altere en general su calidad.

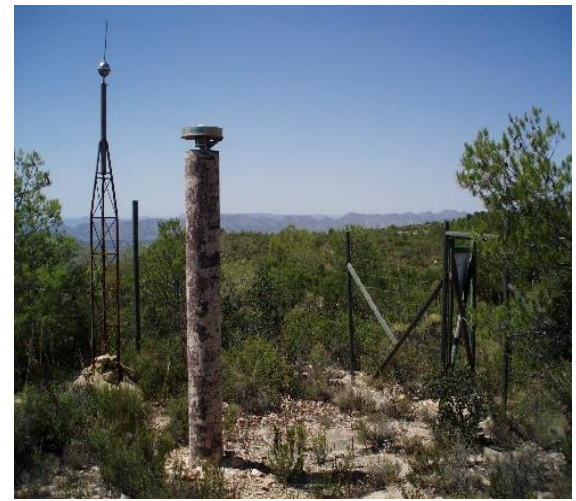

Figura 11. Estación GPS permanente PILA en Sierra de la Pila, Abarán Murcia

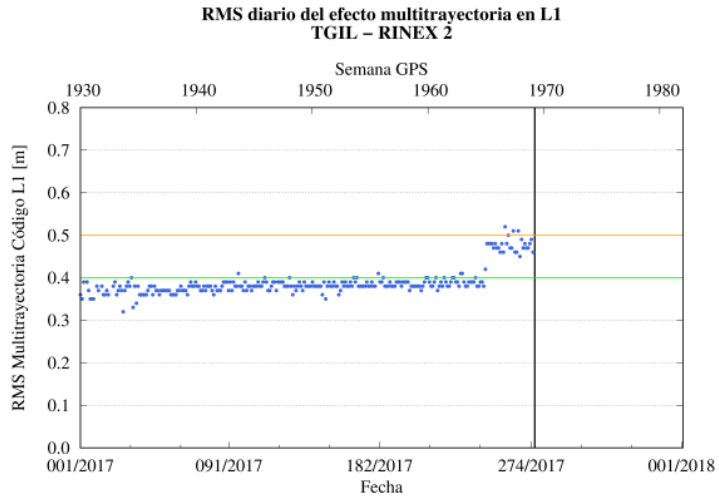

Figura 12. RMS del efecto multitrayectoria en la frecuencia GPS L1 registrada por TGIL durante 2017. Línea vertical negra indica fecha en que se desmanteló la estación

\subsubsection{Estación TGIL}

La gráfica de calidad histórica (Figura 6.E) muestra que el número de observaciones registradas durante su periodo de actividad ha sido el esperado a excepción de algunos periodos invernales, durante los cuales decrecen el número de observaciones y el ratio o/e. Ese comportamiento es similar al observado en la estación PALM y, a excepción de esas anomalías en periodo invernal, el registro de observaciones junto al resto de parámetros se puede 
considerar que están dentro de los valores umbrales de calidad de las estaciones IGS. Basándose en la experiencia con el resto de las estaciones, los periodos de mal funcionamiento en invierno se relacionan con problemas de batería (se reduce su recarga a través de los paneles solares siendo insuficientes las horas de actividad solar, disminuye su capacidad a bajas temperaturas y se pone de manifiesto su antigüedad). Sin embargo, se produjo un brusco deterioro de todos los parámetros en septiembre de 2017 indicando que algo había alterado el entorno de la estación, además del supuesto problema de batería. En su gráfica de calidad anual (Figura 12) se observa claramente ese brusco cambio en el efecto multitrayectoria correspondiendo al DOY 248, que de igual modo se produce en el resto de parámetros de calidad. Finalmente se comprobó que dos grandes depósitos se ubicaron junto a la antena afectando al sector suroeste (Figura 13.B).

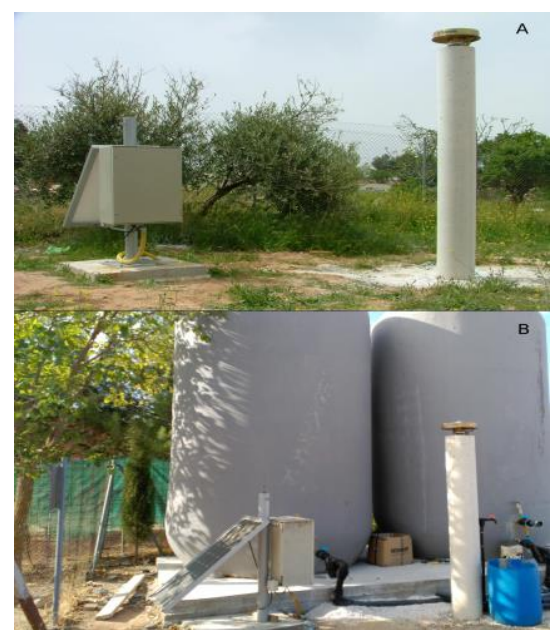

Figura 13. Estación GPS permanente TGIL en Torreperogil, Jaén. A: Aspecto de la estación y su entorno cuando se activó en abril de 2008. B: Aspecto en octubre de 2017, se aprecian algunos obstáculos que afectan a la calidad de las observaciones

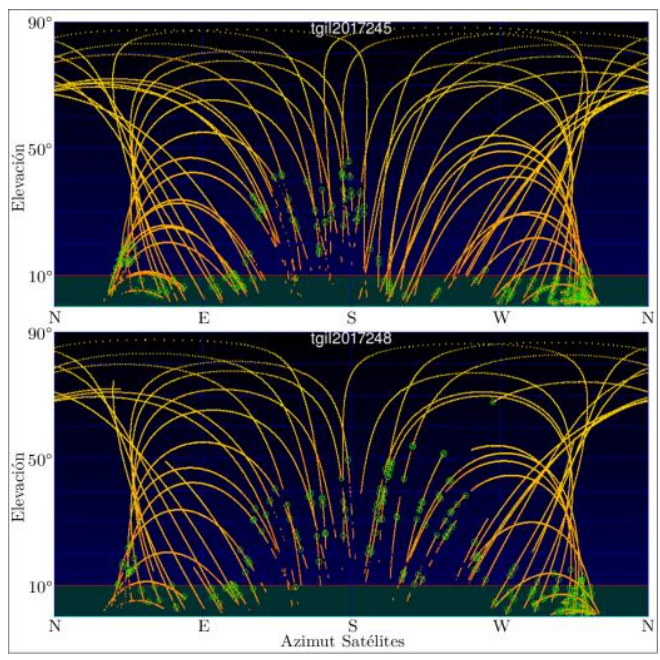

Figura 14. Proyecciones cartesianas de las coordenadas horizontales de los satélites seguidos por la estación TGIL durante los DOY 245 y 248 en 2017. Saltos de ciclo representados con puntos verdes

Para determinar si el brusco deterioro de la calidad estaba relacionado con los depósitos instalados, se comprobó si había cambios significativos en los gráficos del cielo durante los días en los que se inició ese agravamiento. La Figura 14 muestra las proyecciones cartesianas de las coordenadas horizontales de los satélites observados (azimut versus elevación). El 2 de septiembre de 2017 - DOY 245 comenzaron a producirse numerosos saltos de ciclo entre las elevaciones $10^{\circ}$ y $50^{\circ}$ evidenciando oclusiones u obstáculos en el horizonte que provocaban la pérdida de señal. En el DOY 248 aumentó el rango en azimut y elevación en el que se producían los saltos de ciclo, y 
además ese estado del gráfico del cielo se mantuvo constante para el resto de días, por lo que se deduce que los depósitos habían sido ya instalados. Es posible que la instalación de los depósitos comenzara en el DOY 245, por lo que no es recomendable usar los datos a partir de esa fecha.

Independientemente del aumento o disminución del número de observaciones registradas, el efecto multitrayectoria y los saltos de ciclo aumentaron progresivamente a lo largo de los años, apreciándose picos en los saltos de ciclo durante los periodos de primavera-verano. La estación se instaló en terreno privado en 2008, siendo entonces el entorno despejado, salvo algún pequeño árbol que en principio no afectaba al panel solar y situado además por debajo del horizonte de antena (Figura 13.A). El entorno de la estación fue cambiando con el paso del tiempo y en la imagen B se puede distinguir algún árbol muy próximo produciendo sombra sobre el panel solar y claramente por encima del horizonte de antena. En Pirti (2008) se realizó un estudio donde determina el efecto que provocan obstrucciones arbóreas sobre el efecto multitrayectoria, precisión y exactitud de posiciones GPS que empeoran conforme se reduce la distancia a la antena. En Uzodinma y Nwafor (2018) también se confirmó la degradación que pueden producir las copas de los árboles sobre la precisión de la señal GNSS. Se desconoce cuándo ese árbol fue plantado junto a la estación, pero su proximidad, crecimiento y posible aumento de frondosidad en primavera-verano pueden estar relacionados con el aumento de los saltos de ciclo y del efecto multitrayectoria registrados desde 2012. La estación TGIL se desmanteló el 4 de octubre de 2017 - DOY 277 (indicado con línea vertical negra en las gráficas de calidad).

\subsubsection{Estación VILA}

La estación (Figura 15) estuvo activa tan sólo un año, ya que sufrió ataques vandálicos, y se desmanteló el 6 de septiembre de 2009 - DOY 249. A pesar de que sus parámetros de calidad están al nivel de las estaciones IGS de modo similar que AREZ y PILA, un año de observaciones no es suficiente para incluirla en estudios geodinámicos.

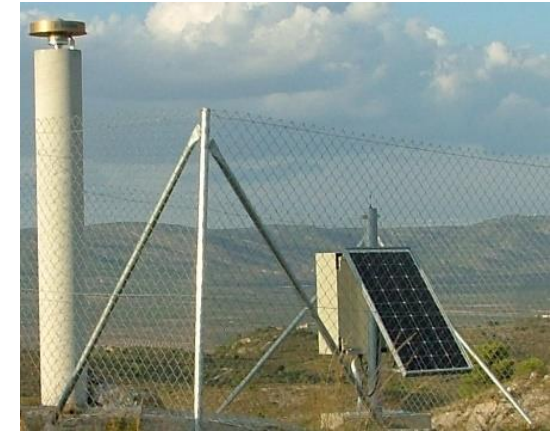

Figura 15. Estación GPS permanente VILA en Sierra de la Villa, Alicante 
Una visión global del estado y calidad de la red Topo-Iberia-UJA desde su activación se muestra en la Figura 16. Se indica con una escala cromática el número máximo de observaciones registradas por 19124 ficheros RINEX v2.11, que suponen la totalidad de los datos disponibles hasta la fecha de control, 31 de marzo de 2019. Se pueden identificar los periodos con ficheros incompletos o perdidos y que las estaciones no han tenido interrupciones notables.

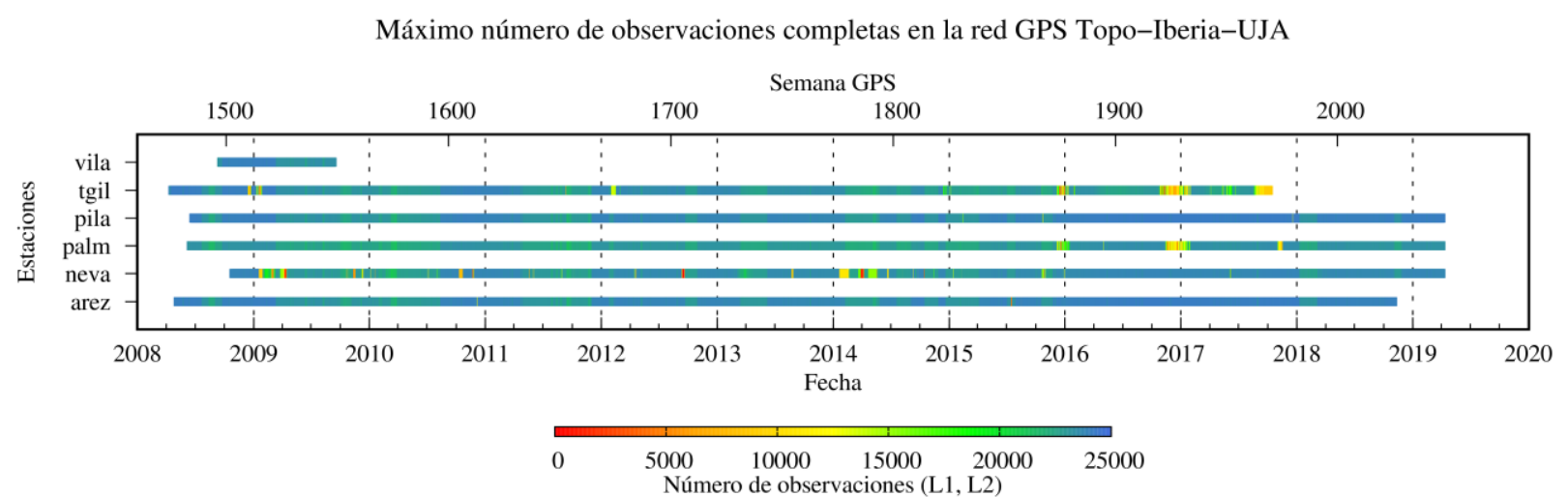

Figura 16. Número de observaciones en los ficheros RINEX v2.11 a lo largo del periodo de actividad de las estaciones de Topo-Iberia bajo el control de la UJA

\section{Conclusiones}

En este trabajo se ha presentado por primera vez el control de calidad de las estaciones cGPS de la red Topo-Iberia que están a cargo de la Universidad de Jaén. En general las estaciones no han presentado valores anómalos para el efecto multitrayectoria y SNR que indiquen un posible problema en sus ubicaciones, presentando además un horizonte de oclusión por debajo de los $10^{\circ}$. Este control de calidad ha puesto de manifiesto que sus emplazamientos son óptimos para conformar una red con fines geodésicos. Para el control de los datos se ha tomado como indicadores de la calidad a los parámetros obtenidos con TEQC. Se ha considerado como referencia o primer nivel de calidad a los valores registrados por la mitad de las estaciones IGS en dichos indicadores. A excepción de los eventos acontecidos en la estación TGIL y de alguna incidencia puntual que fue corregida en NEVA y PALM, se concluye que las estaciones de la red Topo-Iberia-UJA han tenido gran estabilidad y calidad a lo largo de su periodo de actividad y los valores de sus parámetros analizados se corresponden con el primer nivel de calidad de las estaciones IGS.

Se ha descrito la automatización de tareas y procesos a los que son sometidos los datos: descarga desde las estaciones desplegadas en campo, conversión, preprocesado, control de calidad, creación de gráficas y envío al 
IGME. Ese control diario permite monitorizar y detectar posibles problemas en su equipamiento y entorno, ayudando en la toma de decisiones y perder así el mínimo de datos posible.

El IGME recopila los datos de Topo-Iberia y los pone al servicio de los investigadores a través del sistema SiTopo. El estudio presentado sigue metodologías de centros internacionales responsables de redes GNSS y complementa la labor del IGME proporcionando información sobre sus datos GPS recopilados que hasta ahora no existía. Dicha información ayuda a comprender anomalías y también a evitarlas, útil en otros procesos realizados a partir de esos datos como son el control posicional, análisis de series temporales o determinación de vectores de deformación de la corteza terrestre.

\section{Agradecimientos}

Este estudio ha sido cofinanciado por el Subprograma Personal Técnico de Apoyo 2015 del Ministerio de Economía, Industria y Competitividad (PTA2015-11507-I MINECO) y Universidad de Jaén. Quiero mostrar mi agradecimiento al grupo RNM-282 del Plan Andaluz de Investigación por continuar con el mantenimiento de las estaciones y proporcionar algunas de sus fotos. Estoy especialmente agradecido al Dr. Pedro Alejandro Ruiz Ortiz, director del CEACTierra, por facilitar cuanto soporte técnico ha sido necesario, así como al Dr. Mario Sánchez Gómez y a los revisores por sus comentarios y sugerencias que han mejorado notablemente la calidad de este trabajo.

\section{References:}

1. Amante, C., y Eakins, B. W. (2009). ETOPO1 1 Arc-Minute Global Relief Model: Procedures, Data Sources and Analysis. NOAA technical memorandum NESDIS NGDC-24. National Geophysical Data Center, NOAA. doi: 10.7289/V5C8276M.

2. Borque, M. J., Sánchez-Alzola, A., Martin-Rojas, I., Alfaro, P., Molina, S., Rosa-Cintas, S., Rodríguez-Caderot, G., de Lacy, C., García-Armenteros, J. A., Avilés, M., Herrera-Olmo, A., GarcíaTortosa, F., Estévez, A. y Gil, A. J. (2019). How Much Nubia-Eurasia Convergence Is Accommodated by the NE End of the Eastern Betic Shear Zone (SE Spain)? Constraints From GPS Velocities. Tectonics, 38(5), 1824-1839. doi: 10.1029/2018tc004970

3. Carpentier, G., Bruyninx, C., y Roosbeek, F. (2004). Quality and Latency of the data within the EUREF Permanent Network. Proc. of the EUREF symposium held in Bratislava, Slovakia, 14. Disponible en:http://ftp.epncb.oma.be/_documentation/papers/eurefsymposium2 004/quality_and_latency_of_the_data_within_the_euref_permanent_ network.pdf (Último acceso en 15-01-2020). 
4. Estey, L. H., y Meertens, C. M. (1999). TEQC: The Multi-Purpose Toolkit for GPS/GLONASS Data. GPS Solutions, 3(1), 42-49. doi: 10.1007/PL00012778.

5. Estey, L. H. (2013). Latest version of teqc on-line (15 Mar 2013). Disponible en:

https://postal.unavco.org/pipermail/teqc/2013/001492.html (Último acceso en 07-07-2020).

6. Estey, L. H. (2017). Next official version of teqc on-line (2017 Jun 19). Disponible en:

https://postal.unavco.org/pipermail/teqc/2017/002288.html (Último acceso en 07-07-2020).

7. Estey, L. H. (2018). Helpful tip of week 2000. Disponible en: https://postal.unavco.org/pipermail/teqc/2018/002475.html (Último acceso en 14-11-2019).

8. EUREF (2020). EUREF Permanent GNSS Network. Daily Tracking Performances. Disponible en:

http://www.epncb.oma.be/_networkdata/data_quality/index.php?stati on=ACOR00ESP (Último acceso en 15-01-2020).

9. FSF (2016). Gawk - GNU Project - Free Software Foundation (FSF). Disponible en: https://www.gnu.org/software/gawk (Último acceso en 15-01-2020).

10. Galindo-Zaldivar, J., Gil, A. J., Galdeano, C. S., de Lacy, M. C., García-Armenteros, J. A., Ruano, P., Ruiz, A. M., Martínez-Martos M. y Alfaro, P. (2015). Active shallow extension in central and eastern Betic Cordillera from CGPS data. Tectonophysics, 663, 290-301. doi: 10.1016/j.tecto.2015.08.035.

11. Gallart, J. (2006). Geociencias en Iberia: Estudios integrados de topografía y evolución 4-D. «Topo-Iberia». In Memoria Técnica CONSOLIDER-INGENIO 2010. Disponible en:

http://www.igme.es/TopoIberia/xtra/topoiberia-programme.pdf (Último acceso en 15-01-2020).

12. Gárate, J., Martín Dávila, J., Khazaradze, G., Gil, A. J., Jiménez-Munt, I., Gallastegui, J., Ayala, C., Tellez, J., Rodríguez Caderot, G. y Álvarez Lobato, F. (2008). Despliegue de estaciones GPS permanentes en el marco del proyecto Topo-Iberia. $6^{\mathrm{a}}$ Asamblea Hispano Portuguesa de Geodesia y Geofísica, 203, 7-8. Disponible en: http://digital.csic.es/handle/10261/23289 (Último acceso en 15-012020).

13. Gárate, J., Martin-Davila, J., Khazaradze, G., Echeverria, A., Asensio, E., Gil, A. J., de Lacy, M. C., Armenteros, J. A., Ruiz, A. M., Gallastegui, J., Alvarez-Lobato, F., Ayala, C., Rodríguez-Caderot, G., Galindo-Zaldívar, J., Rimi, A. y Harnafi, M. (2015). Topo-Iberia 
project: CGPS crustal velocity field in the Iberian Peninsula and Morocco. GPS Solutions, 19(2), 287-295. doi: 10.1007/s10291-0140387-3.

14. García-Armenteros, J. A. (2010). Estudio de deformaciones de la Corteza Terrestre en la Península Ibérica (Trabajo de investigación conducente al DEA). Universidad de Jaén, España.

15. Hatanaka, Y. (2008). A Compression Format and Tools for GNSS Observation Data. Bulletin of the Geographical Survey Institute, 55, 21-30. Disponible en:

http://www.gsi.go.jp/ENGLISH/Bulletin55.html;

http://terras.gsi.go.jp/ja/crx2rnx.html (Último acceso en 15-01-2020).

16. IGS (2011). Instructions for filling out IGS site logs (April 2011). Disponible en:

https://kb.igs.org/hc/en-us/articles/204197113-Instructions-forfilling-out-IGS-site-logs-April-2011- (Último acceso en 07-07-2020).

17. IGS (2017). Questions about the data quality graphs. Disponible en: https://kb.igs.org/hc/en-us/articles/204229743-Questions-about-thedata-quality-graphs (Último acceso en 15-01-2020).

18. Jaldehag, R. T. K., Johansson, J. M., Davis, J. L., y Elosegui, P. (1996). Geodesy using the Swedish Permanent GPS Network: Effects of snow accumulation on estimates of site positions. Geophysical Research Letters, 23(13), 1601-1604. doi: 10.1029/96GL00970.

19. Jannesen, P. (2016). Ncompress: a fast, simple LZW file compressor. Disponible en: https://github.com/vapier/ncompress (Último acceso en 15-01-2020).

20. Larson, K. M., y Nievinski, F. G. (2013). GPS Snow Sensing: Results from the EarthScope Plate Boundary Observatory. GPS Solutions, 17(1), 41-52. doi: 10.1007/s10291-012-0259-7.

21. Lukyanov, A. V. (2016). LFTP - File transfer program. Disponible en: https://lftp.yar.ru (Último acceso en 15-01-2020).

22. Palano, M., González, P. J., y Fernández, J. (2013). Strain and stress fields along the Gibraltar Orogenic Arc: Constraints on active geodynamics. Gondwana Research, 23(3), 1071-1088. doi: 10.1016/j.gr.2012.05.021.

23. Pirti, A. (2008). Accuracy analysis of GPS positioning near the forest environment. Croatian Journal of Forest Engineering, 29(2), 189-199. Disponible en: http://hrcak.srce.hr/file/51193 (Último acceso en 1501-2020).

24. PSF (2018). Python Software Foundation. Python Language Reference, version 2.7 Disponible en: https://www.python.org (Último acceso en 15-01-2020). 
25. Ramey, C. (2020). Bash, the bourne-again shell. Disponible en: http://tiswww.case.edu/php/chet/bash/bashtop.html (Último acceso en 15-01-2020).

26. Roggero, M. (2010). QC2SKY - TEQC skyplot. Disponible en: https://sites.google.com/site/roggeroresearch/home/software/qc2sky (Último acceso en 15-01-2020).

27. SPI (2020). Debian - The Universal Operating System - Software in the Public Interest (SPI). Disponible en: https://www.debian.org (Último acceso en 15-01-2020).

28. Trimble (2016). Trimble runpkr00 Latest Versions. Disponible en: https://kb.unavco.org/kb/article/trimble-runpkr00-latest-versions744.html (Último acceso en 15-01-2020).

29. UNAVCO (2020). Network Monitoring - PBO GNSS/GPS Network Health. Disponible en:

https://www.unavco.org/instrumentation/networks/status/pbo/health/ AB01 (Último acceso en 15-01-2020).

30. Uzodinma, V. N., y Nwafor, U. (2018). Degradation of GNSS Accuracy by Multipath and Tree Canopy Distortions in a School Environment. Asian Journal of Applied Sciences, 6(4), 214-224. Disponible

en: https://ajouronline.com/index.php/AJAS/article/view/5458 (Último acceso en 15-01-2020).

31. Vaclavovic, P., y Dousa, J. (2016). G-Nut/Anubis - open-source tool for multi-GNSS data monitoring with a multipath detection for new signals, frequencies and constellations. In International Association of Geodesy Symposia Series, Springer, 143, 775-782. doi: 10.1007/1345_2015_97.

32. Wessel, P., Smith, W. H. F., Scharroo, R., Luis, J., y Wobbe, F. (2013). Generic Mapping Tools: Improved Version Released. EOS, Transactions American Geophysical Union, 94(45), 409-410. doi: 10.1002/2013EO450001.

33. Williams, T., y Kelley, C. (2016). Gnuplot - A portable, multiplatform, command-line driven graphing utility. Disponible en: https://sourceforge.net/projects/gnuplot (Último acceso en 15-012020). 